\title{
Molecular-dynamics simulation-based cohesive zone representation of intergranular fracture processes in aluminum
}

\author{
V. Yamakov ${ }^{1 *}$, E. Saether ${ }^{2}$, D. R. Phillips ${ }^{3}$, and E.H. Glaessgen ${ }^{2}$ \\ ${ }^{1}$ National Institute of Aerospace, Hampton, VA 23666, USA \\ 2 NASA Langley Research Center, Hampton, VA 23681, USA \\ ${ }^{3}$ Lockheed Martin Space Operations, Hampton, VA 23681, USA
}

\begin{abstract}
A traction-displacement relationship that may be embedded into a cohesive zone model for microscale problems of intergranular fracture is extracted from atomistic molecular-dynamics simulations. A molecular-dynamics model for crack propagation under steady-state conditions is developed to analyze intergranular fracture along a flat $\Sigma 99\left[\begin{array}{lll}1 & 1 & 0\end{array}\right]$ symmetric tilt grain boundary in aluminum. Under hydrostatic tensile load, the simulation reveals asymmetric crack propagation in the two opposite directions along the grain boundary. In one direction, the crack propagates in a brittle manner by cleavage with very little or no dislocation emission, and in the other direction, the propagation is ductile through the mechanism of deformation twinning. This behavior is consistent with the Rice criterion for cleavage vs. dislocation blunting transition at the crack tip. The preference for twinning to dislocation slip is in agreement with the predictions of the Tadmor and Hai criterion. A comparison with finite element calculations shows that while the stress field around the brittle crack tip follows the expected elastic solution for the given boundary conditions of the model, the stress field around the twinning crack tip has a strong plastic contribution. Through the definition of a Cohesive-Zone-Volume-Element - an atomistic analog to a continuum cohesive zone model element - the results from the molecular-dynamics simulation are recast to obtain an average continuum traction-displacement relationship to represent cohesive zone interaction along a characteristic length of the grain boundary interface for the cases of ductile and brittle decohesion.
\end{abstract}

Keywords: Crack-tip plasticity; Cohesive zone model; Grain boundary decohesion; Intergranular fracture; Molecular-dynamics simulation

*Corresponding author: Tel.: +757-864-2850; fax: +757-864-8912.

E-mail address: yamakov@,nianet.org (V. Yamakov) 
To be submitted to: Journal of the Mechanics and Physics of Solids

\section{Introduction}

Cohesive zone models (CZMs) approximate traction-displacement relationships along an interface (Tvergaard and Hutchinson, 1992; Costanzo and Allen, 1995; Camacho and Ortiz, 1996; Klein and Gao, 1998) and are frequently used in conjunction with the finite element method (FEM) to study fracture in a wide variety of materials. Idealized traction-displacement behavior of interface debonding is embedded into CZM elements. In fracture studies at the micromechanical level, CZM elements can be placed between the continuum finite elements that discretize the grain interior to predict transgranular fracture or placed between the continuum finite elements on either side of a grain boundary to predict intergranular fracture.

Modeling of material failure with CZMs has been advanced to the level of being able to perform large scale simulations of fracture in polycrystals. Recently, Zavattieri et al. (2001) studied the fracture of alumina-ceramic microstructures subjected to multi-axial dynamic loading. The effective size of the polycrystalline specimen studied was $0.54 \times 0.19 \mathrm{~mm}$, thus reaching macroscopic scales. A bilinear traction-displacement relationship parameterized to empirical data, such as macroscopic fracture toughness $K_{I C}$ was used. Zavattieri and Espinosa (2003) used a modification of the same model to study interface effects of an alumina specimen in contact with steel plates. Wei and Anand (2004) have used a modified CZM model to study intergranular fracture in nanocrystalline $\mathrm{Ni}$. In their finite element (FE) model simulation, the CZM element approximated both reversible and irreversible inelastic sliding-separation deformations at the grain boundaries prior to failure. The parameterization of the model was, again, performed by using available experimental data for stress-strain curves of nanocrystalline $\mathrm{Ni}$ in tension with an average grain size of $15-40 \mathrm{~nm}$ and having a comparatively large number of grains. Iesulauro et al. (2002) have applied the CZM technique to simulate fatigue crack 
To be submitted to: Journal of the Mechanics and Physics of Solids

initiation in $\mathrm{Al}$ polycrystals. The use of $\mathrm{MD}$ simulations to parameterize the tractiondisplacement curve was suggested, but the actual parameterization was performed by using the established macroscale yield properties of aluminum.

The macroscale values of strength and toughness that are input to the CZM in these references represent the aggregate responses of thousands or millions of grains, grain boundaries, and defects within the specimens from which they were obtained. Thus, these macroscale values do not represent the unique response of a particular interface at which a local fracture event might occur. If the microscale predictions are to become quantitative, consideration of the local nanoscale properties is required. One possible means of making this connection is to use the results of atomistic MD models as input to the CZM. This connection would allow more realistic simulations leading to accurate predictions of the failure properties of a large class of materials and microstructures, even when experimental data is not available.

Attempts to extract relevant parameters for the decohesion law of a CZM from atomistic (molecular-dynamics or molecular-static) simulations have been made by various groups in the last few years (Gall et al., 2000; Komanduri et al., 2001; Spearot et al., 2004). The approach in all of these works is based on simulating the debonding of a flat interface under a constant tensile strain rate perpendicular to the interface. In these references, the system size is between 4 and 8 $\mathrm{nm}$, and the dynamics of the atoms is severely constrained by the boundary conditions, which do not allow for Poisson lateral contraction and shear deformation. As a result, plastic processes, such as dislocation slip, are strongly suppressed. Consequently, the simulated mechanism for interface decohesion in these references reproduces the process of atomic adhesion (strength) rather than that of fracture at the interface. Raynolds et al. (1996) used a similar setup to study adhesion in an NiAl-Cr interface by first principles calculations. 
To be submitted to: Journal of the Mechanics and Physics of Solids

The boundary conditions, at which a typical CZM element operates in a large scale FE model are very different from the ones used in the referenced MD and first principles simulations. Typically, the CZM elements are embedded within a system of finite elements, that reproduce the elastic and plastic response of the surrounding material to both the external load and the crack-tip stress. In contrast, decohesion parameters, such as peak stress and opening displacement of the CZM curve, are extracted from an atomistic volume less than $10 \mathrm{~nm}$ in each direction. The lack of an adequate surrounding volume of material suppresses the plastic processes, such as dislocation nucleation, limiting the accommodation of deformation at the interface and forcing it to debond in an unnatural manner. The periodic boundary conditions usually applied in these models cause the simulation to create a response of an array of repeating units with a strong overlap of image elastic forces rather than the response of a single specimen unit. Consequently, the resulting decohesion curves cannot be directly applied to derive the constitutive laws for CZM elements.

The main goal of the approach described in the present study is to extract, and understand the contributions to, an MD-based CZM decohesion law for intergranular fracture under local conditions similar to those experienced by the CZM element in a polycrystalline FE model. The CZM decohesion law reflects the response of the CZM element to an approaching and propagating crack (Costanzo and Allen, 1995; Dávila, 2001). Thus, the MD model should be a model of crack propagation rather than of adhesion. The MD model used in this study is built to simulate a crack propagating through a flat high-energy grain-boundary in aluminum (Yamakov et al., 2005).

The paper is constructed as follows: The simulation approach is described in Section 2. The mechanism of intergranular crack growth together with the plastic processes near the crack tip as 
To be submitted to: Journal of the Mechanics and Physics of Solids

revealed by $\mathrm{MD}$ simulations at the atomic level is broadly discussed in Section 3 . The contribution of the plastic processes to the stress field near the crack tip is discussed in Section 4. Section 5 describes a methodology for extracting a constitutive relation for a continuum CZM element from the MD results. The main conclusions of this study are outlined in Section 6.

\section{The simulation approach}

The simulation approach used in this study is based on a MD simulation model of crack propagation under time-independent, or steady-state, conditions through a flat grain boundary (GB) in Al (modeled by the interatomic potential of Mishin et al. (1999)) at low temperature $(100 \mathrm{~K})$. The purpose of the MD simulation is to reveal and analyze the atomistic processes taking place near the crack tip and to derive a statistical traction-displacement relationship for a continuum CZM element. The simulation must also provide a study on the influence of the atomistic processes on the resulting traction-displacement (decohesion) curve.

To produce reliable and time-independent statistics for extracting the CZM decohesion law the simulation model is based on a well known continuum-elastic analytical model for steadystate crack propagation, which is briefly reviewed in Section 2.1. Following the analytical model, the MD set up and the details of the MD simulation are discussed in Section 2.2. Major attention is given to the crystallography of the atomistic MD system, which determines the behavior of the model and, to a large extent, predefines the ongoing atomistic processes.

The values of the initial and the simulation parameters of the MD system are crucial for recovering the steady-state regime in the MD simulation. To set up these parameters, specifically the system size, the initial crack length and the initial loading conditions, a continuum elastic solution of the system is needed. This solution cannot be taken from the analytical model, which 
To be submitted to: Journal of the Mechanics and Physics of Solids

has an infinite dimension (see Section 2.1), because the finite size effects always present in an MD simulation make it impossible for the MD system to reproduce exactly the analytical model. In this work, a linear elastic solution is obtained by performing a FE simulation on an elastically equivalent continuum system (described in Section 2.3), and provides for a direct comparison between the MD and the FE results. Such a comparison helps to distinguish the role of the plastic processes such as dislocation nucleation, vacancy and void formation, etc. present in the MD simulation, but not in the FE simulation.

\subsection{The analytical model}

The theoretical model for steady-state crack propagation adapted and used in this work is discussed and analyzed by Langer and co-authors (Barber et al., 1989; Langer, 1992; Langer, 1993; Langer and Nakanishi, 1993). The model represents a laterally strained strip of elastic material. The strip is infinite in the longitudinal direction, and a semi-infinite crack propagates through it (Barber et al., 1989). Far ahead of the crack tip, the strip is under uniform tension. Far behind the tip, the tension is relieved, and the crack opening becomes constant. Thus, there are two stable states for the material: "closed", in front of the crack tip, and "open", behind the crack tip. The crack propagation can be viewed as a continuous steady-state transition between these two states (Barber et al., 1989). The stress field of a steadily moving crack for this model was calculated by Ching (1994). Applying this model as a base for the MD simulation (Yamakov et al., 2005) ensures that after some initial unsteady growth, the crack propagation will proceed under steady-state conditions independent of the crack length.

\subsection{The molecular-dynamics model}


To be submitted to: Journal of the Mechanics and Physics of Solids

The MD simulation is based on the previously described analytical model for steady-state crack propagation and has the configuration shown in Fig. 1 (Yamakov et al., 2005). With periodic boundary conditions in all directions, the model represents an aluminum multilayer system of alternating sets of thick and thin crystalline layers separated by four flat GBs. The two broad layers, marked as "Crystal I" and "Crystal II", form a bicrystalline system with a flat GB in the middle, through which the crack propagates. The crystallographic orientations of Crystal I and Crystal II are presented in Fig. 2. In the imposed coordinate system of the model, the orientation of Crystal I is: $\left(\mathrm{x}:\left[\begin{array}{lll}7 & \overline{7} & \overline{10}\end{array}\right]\right.$; $\mathrm{y}:\left[\begin{array}{lll}5 & \overline{5} & 7\end{array}\right]$; $\left.\mathrm{z}:\left[\begin{array}{lll}1 & 1 & 0\end{array}\right]\right)$, and the orientation of Crystal II is: (x:[ $\left.\begin{array}{lll}7 & \overline{7} & -\overline{0}\end{array}\right]$; $\mathrm{y}:\left[\begin{array}{lll}\overline{5} & 5 & \overline{7}\end{array}\right]$; $\left.\mathrm{z}:\left[\begin{array}{lll}1 & 1 & 0\end{array}\right]\right)$ (see Fig. 2). In this way, Crystal II is a mirror image of Crystal I relative to the crystallographic plane $\left\{\begin{array}{lll}5 & 5\end{array}\right\}$, which becomes the plane of the GB between them. The GB thus formed is a $<110>\sum 99$ symmetric tilt GB, for which the atomic structure in $\mathrm{Al}$ is known from the literature (Dahmen, 1990). This is a highangle grain boundary (tilt angle of $89.42^{\circ}$ ) with a large excess (i.e., above the perfect crystal) energy, $\gamma_{\mathrm{GB}}=0.60 \pm 0.05 \mathrm{~J} / \mathrm{m}^{2}$, estimated here for a relaxed structure at $\mathrm{T}=100 \mathrm{~K}$. The high excess GB energy facilitates its decohesion (Wolf, 1990). The surface energy of the GB plane $\gamma_{\mathrm{s}}$ at $100 \mathrm{~K}$ is estimated in this work at $\gamma_{\mathrm{s}}=0.952 \pm 0.010 \mathrm{~J} / \mathrm{m}^{2}$ and is in good agreement with experimental data for the Al surface energy.

The two smaller layers, Absorbing Layer I and Absorbing Layer II, on both sides of the bicrystalline system (Fig. 1) have the same crystallographic orientations as Crystal II and Crystal I, respectively. Consequently, the GBs formed by these layers are of the same crystallographic type as the GB between Crystal I and Crystal II. The purpose of these layers in the simulation is to serve as shock-wave absorbers (Gumbsch, 1997), where a damping friction coefficient is applied to the atoms to absorb the phonon waves generated from the crack tips. In addition, the 
To be submitted to: Journal of the Mechanics and Physics of Solids

GBs between these layers and Crystal I and II act as absorbers for the dislocations that may be emitted and spread out from the crack tip during propagation. In this way, the negative effect of the periodic boundary conditions in the $y$-direction creating periodic images of all crack-tip disturbances and influencing the crack propagation is suppressed.

The interaction between individual atoms in the system is presented by a many-body embedded-atom method (EAM) potential of Mishin et al. (1999) fitted to give the correct zerotemperature lattice constant, $\mathrm{a}_{\mathrm{o}}=4.05 \AA$, elastic constants, cohesive energy, vacancy formation energy, etc. Of particular importance for the simulation of fracture and dislocation plasticity is the close fit of the potential to the experimentally measured surface and stacking-fault energies. Potential-dependent parameters that will be needed in this study are the relaxed stable stackingfault energy, $\gamma_{\mathrm{sf}}=0.146 \mathrm{~J} / \mathrm{m}^{2}$, the unstable stacking-fault energy, $\gamma_{\mathrm{us}}=0.168 \mathrm{~J} / \mathrm{m}^{2}$, as defined in Mishin et al. (1999), and the unstable twinning energy, $\gamma_{\mathrm{ut}}=0.210 \pm 0.010 \mathrm{~J} / \mathrm{m}^{2}$ estimated here according to the method described in Tadmor and Hai (2003).

The system thickness $h$ in the $z$-direction equals only $10\left(\begin{array}{lll}2 & 2 & 0\end{array}\right)$ crystallographic planes (accounting for the symmetry of the fcc lattice), or $h=10 \sqrt{ } 2 / 2 \mathrm{a}_{\mathrm{o}} \approx 2.9 \mathrm{~nm}$. This thickness is more than four times larger than the range of the interatomic potential, $\mathrm{r}_{\mathrm{c}}=1.55 \mathrm{a}_{\mathrm{o}}=0.628 \mathrm{~nm}($ Mishin et al., 1999), which prevents interference of the atoms with their periodic images and preserves the local three-dimensional (3D) physics in the system. The small thickness in the $z$-direction allows the system size in the $x$ - and $y$-directions to extend up to $21<7710>\mathrm{a}_{\mathrm{o}} \approx 120 \mathrm{~nm}$, and $24<557>\mathrm{a}_{\mathrm{o}} \approx 97 \mathrm{~nm}$, respectively (see Fig 1 and Fig. 2), while limiting the number of simulated atoms to $1,994,000$, allowing the simulation to be carried out on a modest Beowulf cluster.

The choice of the $\left[\begin{array}{lll}1 & 1 & 0\end{array}\right]$ crystallographic orientation of the smallest system dimension $z$ is

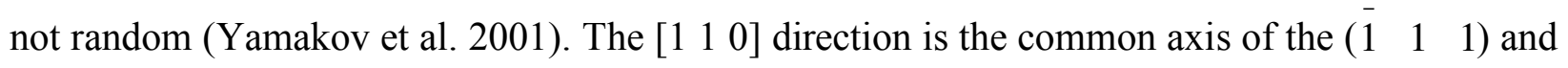


To be submitted to: Journal of the Mechanics and Physics of Solids

(1 $\left.\begin{array}{ll}1 & 1\end{array}\right)$ planes, which are glide planes for the most common slip dislocations (i.e., $1 / 2<110>$ dislocations) in fec metals. It has been shown (Yamakov et al., 2003a) that all of the six slip systems available on these two planes can operate unobstructed by the small system size in this direction. Moreover, a number of complex dislocation interactions and events are still possible between these six slip systems, as in a full 3D space (Yamakov et al., 2002; 2003a). As the dislocation activity is expected to be very important in this study, the choice of the $\left[\begin{array}{lll}1 & 1 & 0\end{array}\right]$ direction as a columnar axis for this quasi-two dimensional set-up ensures the best resemblance to a full $3 \mathrm{D}$ environment, the main constraint being that the dislocation lines of all possible dislocations have to be straight lines parallel to the columnar direction. A comparison between this quasi-2D and a full 3D environment in a nanocrystalline model shows that some grain size effects, related to the curvature of the dislocation loops presented in $3 \mathrm{D}$, are suppressed (Yamakov et al., 2003b). Specifically, as the stress needed to expand a dislocation loop is inversely proportional to the loop radius, which, by itself, is limited by the very small grain size of the nanocrystalline metal, there is a substantial grain size dependence of the yield stress. This geometrical dependence is absent in a quasi-2D model, because the small thickness of the system constrains the dislocation lines to be always straight and parallel to each other preventing the formation of dislocation loops. The lack of curvature of the dislocation lines, as well as of the crack front, should also be taken into account as a simplification in this model. In the case where the grain size is large enough not to be a governing parameter, the presence of curvature usually helps the defect nucleation process at or around the crack tip, such as dislocations and microvoids. While it is expected that neglecting the effects of curvature would not qualitatively change the fracture mechanism, it may affect the process quantitatively in terms of slightly decreased peak stress and work of debonding. 
To be submitted to: Journal of the Mechanics and Physics of Solids

The simulations are run at a temperature of $\mathrm{T}=100 \mathrm{~K}$, which is low enough to suppress GB and surface diffusion processes and to facilitate brittle fracture in aluminum. The temperature is kept constant by using the Nose-Hoover thermostat (Nose, 1984).

The system is driven to the initial condition of the simulation before crack nucleation in several steps. First, the system is constructed out of four blocks of perfect crystals (each one for Crystal I, Crystal II, Absorbing Layer I, and Absorbing Layer II in Fig. 1), which have been independently thermally equilibrated at $100 \mathrm{~K}$ at zero constant pressure. The constant pressure equilibration is achieved by using the Parrinello-Rahman constant-stress simulation (Parrinello and Rahman, 1981). When the perfect crystals are joined together into a multilayer system to form $\Sigma 99 \mathrm{GBs}$, they are also allowed to shift along the GB planes (in the $x$ - direction) to obtain the lowest energy initial GB configuration. After being assembled, the whole system is equilibrated at $100 \mathrm{~K}$ and zero pressure to achieve thermal equilibrium of the GB interfaces. Because the building crystal blocks have been already preheated to $100 \mathrm{~K}$, the thermalization of the whole system takes much less time than if it were built out of ideal crystals at $0 \mathrm{~K}$ and then thermalized.

After the thermal equilibration at zero pressure, the system is loaded hydrostatically in tension, i.e.,

$$
\sigma_{x x}=\sigma_{y y}=\sigma_{z z}=\sigma
$$

and is dynamically equilibrated at this constant stress. After establishing equilibrium between the strain in the system and the applied external stress, the system size in all three dimensions is fixed under the constant strained condition.

The transition from a constant stress to a constant strain simulation transforms the volume fluctuations, always present in a finite system under thermodynamic equilibrium, into stress 
To be submitted to: Journal of the Mechanics and Physics of Solids

fluctuations. Thus, further equilibration at constant strain is necessary to smooth out these fluctuations. The simulation then proceeds under this constant strain - constant temperature condition. Although the simulations are carried out under constant strain, for convenience in presentation, the various analyses will reference the value of prestresses that corresponds to a particular value of prestrain.

To ensure crack nucleation and growth at the nanometer scale, the prestress was varied between 3.5 and $4.25 \mathrm{GPa}$. If the system were uniaxially strained, these very high prestress values would have caused strong plasticity effects not related to the crack, such as spontaneous dislocation nucleation from the GBs (Yamakov et al., 2002). Applying triaxial hydrostatic stress eliminates these undesirable plasticity effects.

The crack in the system is nucleated by screening (preventing) the atomic interactions between atoms at both sides of the GB plane between Crystal I and Crystal II along a region of length $l_{\mathrm{o}}$. As the crack grows and the crack opening becomes large enough to prevent interaction of atoms on the adjacent crack faces along the screened region, the previously screened atomic interactions are restored and the crack continues to evolve on its own. The crack starts growing if $l_{\mathrm{o}}$ is larger than the critical Griffith length $L_{g}$ defined when the energy spent to create the upper and lower crack surface $2 \gamma_{\mathrm{s}}$ minus the energy gained by destroying the GB $\gamma_{\mathrm{GB}}$ is equal to the released strain energy $-d U / d l$, per length $l$,

$$
2 \gamma_{\mathrm{s}}-\gamma_{\mathrm{GB}}=-d U / d l
$$

An estimate of $L_{g}$ is made by calculating $d U / d l$ as a function of $\sigma$ and $l$. This calculation is performed by using an anisotropic elastic finite element model of the elastic equivalent of the MD system, as will be discussed later. For the values of prestress applied in this study, $\sigma=3.5$, 3.75, 4.0, and 4.25 GPa, the obtained Griffith lengths are $L_{g}=6.08,5.32,4.71$, and $4.21 \mathrm{~nm}$, 
To be submitted to: Journal of the Mechanics and Physics of Solids

respectively. The initial crack length is set equal to the crystallographic period in the $x$-direction, $l_{\mathrm{o}}=<7710>a_{\mathrm{o}}=5.7 \mathrm{~nm}$, to reflect the existing periodicity of the equilibrated GB structure (Dahmen, 1990) induced by the lattice of the two joined crystals. This value of $l_{\mathrm{o}}$ is larger than $L_{g}$ for most of the prestresses, except for $\sigma=3.5 \mathrm{GPa}$, ensuring the initiation of crack growth for these prestresses. Crack growth also occurs for $\sigma=3.5 \mathrm{GPa}\left(L_{g}=6.08 \mathrm{mn}\right)$, because the use of a many-body potential causes all the atoms within the interaction range of the screened atoms to be also affected by the screening, effectively increasing the initial crack length.

The identification of various structural defects including dislocations, twins, stacking faults, etc. appearing around the growing crack is important for understanding the mechanism of deformation that influence the CZM constitutive law. A procedure for atom identification based on the atom's coordination number and on the common-neighbor-analysis (CNA) technique (Honeycutt and Andersen, 1987; Clarke and Jonsson, 1993) is used. The technique makes it possible to identify atoms in fcc and hep states. Layers of hcp atoms in an fcc lattice are formed at stacking faults and twin boundaries (Weertman and Weertman, 1992) and can be used very successfully for visualizing the ongoing dislocation processes in fcc crystals (Schiotz, 1999). Atoms that are not identified in an fcc or hcp state are considered to be in a non-crystalline state and indicate the presence of GBs or dislocation cores. In addition, atoms that have lost more than $1 / 3$ of their neighbors inside the interaction range of the potential are considered surface atoms. On average, $1 \mathrm{~nm}^{2}$ of a flat $\{557\}$ surface contains 16 surface atoms. The crack free surface $S$ is estimated by counting the number of surface atoms, and when divided by $2 h$ (see Fig. 1 , the prefactor 2 accounts for the two crack surfaces), conveniently gives an effective crack length $l$. Under this convention, the thermalized structure of a $\langle 110\rangle \Sigma 99$ symmetric tilt GB, shown in Fig. 2, appears as quasi-periodic, with a regular pattern of hcp atoms immersed in a disordered layer 
To be submitted to: Journal of the Mechanics and Physics of Solids

with the presence of a few distributed vacancies, identified by the appearance of a few isolated surface atoms inside the GB region. As will be shown later in this paper, classifying atoms in this way presents a unique possibility to distinguish and quantify the various atomic processes occurring at the crack tip, and to identify their contribution to the decohesion law of the CZM elements.

\subsection{The finite element model}

A FE configuration, with dimensions scaled to reproduce the proportions of the MD system, is presented in Fig. 3. The FE calculations are anisotropic linear-elastic, carried out under displacement control in the $x$ - and $y$-directions, and use generalized plane strain to reproduce the hydrostatic triaxial stress in the MD simulation. The use of periodic boundary conditions in the constant strain MD simulation, described previously, constrains the problem in the same manner as the generalized plane strain condition in continuum mechanics. The commercial software package ABAQUS (ABAQUS, 2004) is used. The model contains a combination of six-node triangular elements and eight-node quadrilateral elements that support generalized plane strain. The anisotropic elastic constants are obtained from the MD interatomic potential and transformed according to the crystallographic orientations of the atomic microstructure (see Appendix A). Higher order terms in the elastic constants are not included as they are not available from the published data for this potential (Mishin et al., 1999) and are not easy to calculate. This limits the FE simulation to be linear elastic.

The FE model contains a built-in lenticular slit, which simulates the MD approximation to a crack of varying relative length, $0.05<l / L<0.91$, and is used to study the evolution of the system at different stages of the crack growth. To avoid stress singularities, the edges of the slit have a finite, but very small, initial radius $\lambda=0.008 L$, which, when scaled to the MD 
To be submitted to: Journal of the Mechanics and Physics of Solids

dimensions, corresponds to an initial crack opening of approximately $1 \mathrm{~nm}$, i.e., slightly larger than the range of the interatomic MD potential.

Two significant differences between the FE and MD models have to be outlined here. First, the FE calculations are static and linear-elastic. No dynamic or nonlinear effects are incorporated, and the results are relevant for static "cracks" under small strain when the nonlinear effects can be neglected. Second, there is no elastic equivalent of the GBs in the FE system. It is assumed that the difference between the elastic response of the GBs and the interior of the grains does not significantly alter the crack behavior because of the relatively small volume ratio of the GB in the system. Thus, the FE simulations used here play mainly a guiding role in setting up the parameters of the MD model and in understanding the MD results by distinguishing between plastic and elastic processes in the MD simulation.

The first implementation of the FE model is to perform energetic analysis of the system in the absence of nonlinear elastic or plastic processes and to extract the Griffith length $L_{g}$ for the different loads of the MD system using Eq. (2). The elastic strain energy per unit thickness, $E / h$, of the FE system versus increasing crack length, $0 \leq l \leq 110 \mathrm{~nm}$, is presented in Fig. 4 . The values for $l=0$ relates to a system with no crack, and is given for reference. Because the FE simulations are linear elastic, the strain energy can be easily scaled to the absolute dimensions of the MD system and to the four different applied prestresses $\sigma$. Knowing $\gamma_{\mathrm{s}}$ and $\gamma_{\mathrm{GB}}$, extracted from the MD model and given in Section 2.2, one can plot their effective contributions to the crack energy as two straight lines: for the surface energy, $E_{\text {surf }}=2 \gamma_{\mathrm{s}} l$; and for the change of the GB energy, $\Delta E_{\mathrm{GB}}=-\gamma_{\mathrm{GB}} l$, as shown in Fig. 4. Summing the elastic energy $E_{\text {elast }}(\sigma, l)$, $E_{\text {surf, }}$ and $\Delta E_{\mathrm{GB}}$ gives the total energy balance of the system for each prestress condition,

$$
E_{\text {tot }}(\sigma, l)=E_{\text {elast }}(\sigma, l)+\left(2 \gamma_{\mathrm{s}}-\gamma_{\mathrm{GB}}\right) l
$$


To be submitted to: Journal of the Mechanics and Physics of Solids

For each $\sigma, E_{\text {tot }}(\sigma, l)$ has a maximum at the Griffith length $l=L_{g}(\sigma)$ as shown in Fig. 4 . The precise values of these maxima are obtained by using second order polynomial interpolation of the FE data points for $E_{\text {elast }}(\sigma, l)$ at $l=0,6$, and $10 \mathrm{~nm}$ in Fig. 4 for each prestress $\sigma$. These results set the initial length, $l_{\mathrm{o}}=5.7 \mathrm{~nm}$, of the nucleated crack in the MD model, as discussed in the previous subsection. More importantly, the FE simulation shows the region for steady-state crack propagation. This steady-state region is where the decrease of $E_{\mathrm{tot}}(\sigma, l)$ with increasing $l$ becomes almost constant. The steady-state region is revealed by drawing a straight, or steady-state, line $(s-$ $s)$ through $E_{\text {elast }}(\sigma, l)$ for $\sigma=4.25 \mathrm{GPa}$ in Fig. 4. The line overlaps with $E_{\text {tot }}$ in the interval $30<l<$ $80 \mathrm{~nm}$ indicating the steady-state region in Fig. 4 . For $L_{g}<l<30 \mathrm{~nm}$, the crack propagation is expected to increase (nucleation region in Fig. 4) because the slope of $E_{\text {tot }}$ increases, approaching the steady-state value. For $l>80 \mathrm{~nm}$, the crack starts to be influenced by the finite length of the system (finite-size effects region in Fig. 4), and $E_{\text {tot }}$ increases above the steady-state value due to the increasing contribution of the crack's periodic image. As the size of the steady-state region is a result of the system dimensions only, it remains the same for all the applied loads in the simulation. Thus, FE simulations help to estimate $L_{g}$ and to find the region of crack lengths where the crack is expected to grow under steady-state conditions, allowing for time independent statistics to be extracted for derivation of the CZM decohesion law.

\section{The mechanisms of crack propagation along the $\Sigma 99$ grain boundary by MD}

\section{simulation}

As explained in Section 2, the GB opens after 8 ps of screening of the atomic interactions in a region of $5.7 \mathrm{~nm}$ length along the middle of the GB between Crystal I and Crystal II (Fig. 1), and a crack starts to grow in both directions along the GB interface. Fig. 5 shows MD snapshots of cracks that have grown for four different initial hydrostatic prestresses: $\sigma=3.5,3.75,4.0$, and 
To be submitted to: Journal of the Mechanics and Physics of Solids

4.25 GPa. In all cases, the crack growth is not symmetric in the $+x$ and $-x$ directions (as defined in Fig. 1) along the GB (1) in Fig. 5). The growth in the $-x$ direction has produced two twin patterns (2) in Fig. 5) by emitting a series of twinning dislocations (3) in Fig. 5) (Weertman and Weertman, 1992), and is almost symmetric about the grain boundary of the two adjacent crystals. The crack propagation in this direction is greatly reduced, when compared to the propagation in the $+x$ direction, with energy expended through ductile blunting at the crack tip. The growth in the $+x$ direction proceeds through a continuous process of void formation (4) in Fig. 5). Because the crack tip emits very few dislocations (3) and (5) in Fig. 5), crack propagation in the $+x$ direction proceeds much faster than in the $-x$ direction, and does so in an almost brittle fashion. After being emitted, some of the dislocations glide away from the crack tip and are absorbed by the next GB layer to form GB dislocations (6) in Fig. 5). Secondary slip (7) in Fig. 5) on a slip plane along the $\left[\begin{array}{lll}1 & \overline{1} & \overline{2}\end{array}\right]$ direction (see Fig. 2) may also accommodate deformation non-parallel to the primary slip plane along the $\left[\begin{array}{lll}1 & \overline{1} & 2\end{array}\right]$ direction, where twinning (3) and primary slip dislocations (5) propagate.

A detailed analysis of all of the atomistic processes, accompanying the crack growth and governing the decohesion of the GB interface follows.

\subsection{Twinning at the crack tip - crack propagation in the $-x$ direction}

Fig. 6 contains a series of snapshots of the crack shown in Fig. 5(c) monitoring the formation of two symmetrical twins at the crack tip propagating in the negative, $-x$, direction in the system (as defined in Fig. 1). Although twinning is not common in Al, twin formation near a crack tip has been reported on several special occasions, both in MD simulations (Farkas et al., 2001; Hai and Tadmor, 2003) and in experiments (Pond and Garcia, 1982; Chen et al., 1999). Twinning has 
To be submitted to: Journal of the Mechanics and Physics of Solids

been found to occur in special cases, when the crack propagation direction is suitably oriented with respect to the available slip planes in the crystal. In these references, the crack propagation is transgranular and the twins are formed along the most energetically favorable slip plane inclined with respect to the crack propagation direction.

As shown in Fig. 6(a), the twinning process starts with a spontaneous emission of $1 / 6$ $\left[\begin{array}{lll}1 & \overline{1} & 2\end{array}\right]$ Shockley partial or twinning dislocations (3) (see, also, (3) in Figs. 5(a-c)) (Weertman and Weertman, 1992) on the $\left(\begin{array}{lll}\overline{1} & 1 & 1\end{array}\right)$ planes in both crystals (see Fig.2 for the crystallographic orientations in the model). Each dislocation creates a stacking fault $(7)$, which is broadened into a nanotwin (2) by the subsequent emission of more twinning dislocations (3) of the same type (Figs. 6(b-f)). Each new dislocation results in the propagation of the crack tip by one atomic layer. If the twinning process starts simultaneously on both, the $-y$ face and the $+y$ face at the $-x$ crack tip, as shown in Fig. 6(a), the two twins grow symmetrically in Crystal I and Crystal II (Figs. 6(b-f)). If the twinning processes do not start simultaneously due to some small perturbations in the simulation, as shown in Fig. 7(a) (to be discussed later), where the twinning in the $-y$ direction (2) is initiated first, the two twins in Crystal I and Crystal II may be offset (Fig. 7(b)). As the crack grows, the symmetry is nearly restored, as can be seen in Fig. 5(b). Thus, the symmetry of the crystal lattice on both sides of the GB leads to a corresponding symmetry in the twin formation, which follows the symmetric orientations of the slip planes relative to the GB.

Secondary slip inside the twins occurs in the form of partial dislocation emission identified by trailing stacking faults ( 7 in Fig. 5(c) and Figs. 6(e,f)). This happens because the growing twin cannot accommodate all of the shear produced at the crack tip. The twinning changes the orientation of the $\left(\begin{array}{lll}1 & 1 & 1\end{array}\right)$ slip plane from $\alpha_{1} \approx 9^{\circ}$ to a new orientation $\alpha_{2} \approx 30^{\circ}$ from the crack plane (Fig. 6(f)). The latter orientation is more favorable for dislocation emission because it 
To be submitted to: Journal of the Mechanics and Physics of Solids

suggests a higher resolved shear stress. Thus, secondary slip is initiated on that plane, giving an additional degree of freedom, which makes accommodation of any deformation in the $(x, y)$ plane near the crack tip possible.

The active twinning through the spontaneous emission of twinning dislocations at the crack tip, together with the development of secondary slip inside the twins, makes the propagation in the $-x$ direction of a ductile type and involves plastic mechanisms that expend considerable energy. The MD simulation results from this crack tip will later be used to construct the cohesive law for a ductile type CZM element.

\section{$3.2 G B$ decohesion by cleavage - crack propagation in the $+x$ direction}

The propagation by cleavage in the $+x$ direction for the crack under a $3.75 \mathrm{GPa}$ prestress is monitored in Fig. 7. First, the disorder of the GB region in front of the crack tip is increased (Fig. 7(a)) until a void appears (4) in Fig. 7(b)). The void grows (4) in Fig. 7(c)) and eventually joins the main crack, increasing its length (Fig. 7(d)). Then, another void forms (4) in Fig. 7(d)) and the process repeats, becoming the primary mechanism for crack propagation. Figure 7(e) shows the formation of two successive voids in front of the crack tip that are about to join to form an increment of crack growth (Fig. 7(f)). A similar mechanism of fracture in an alloy system has been reported in MD simulations of intergranular crack propagation along a high-angle GB in NiAl (Farkas, 2000a; 2000b).

Interestingly, dislocations are not emitted until the final stage of growth (Fig. 7(f)), when the crack is blunted by a spontaneous emission of dislocations (3) and (5) in Fig. 7(f)). The spontaneous dislocation emission is believed to be caused by a dynamic instability at the crack tip that is known to happen at a propagation speed of approximately $1 / 3$ the Rayleigh speed of sound $c_{R}$, and leads to a dynamic brittle-to-ductile transition in the crack propagation (Abraham 
To be submitted to: Journal of the Mechanics and Physics of Solids

et. al., 1994; Abraham, 2001; Gao, 1996; Yamakov et al., 2005). The appearance of the dynamic instability in the theoretical model of Barber et al. (1989), used as a base for the system simulated here (discussed in Sec. 2.1), was predicted by Ching (1994). There, it was stated that steady-state crack propagation above the critical speed is unstable. The MD simulation, in the present work, supports this statement. Additional details are given by Yamakov et al. (2005), wherein the dynamic aspects of crack propagation are discussed.

The lack of dislocation emission from the crack tip propagating in the $+x$ direction localizes the damage zone along the GB interface, where the microvoids are nucleated (Fig. 7(b-e)). The $+x$ propagation is much more brittle and propagates much further along the GB than is observed in the $-x$ direction. The results from this crack tip will later be used to construct the cohesive law for a brittle type CZM element.

\subsection{Rice and Tadmor-Hai criteria for twinning vs. cleavage at the crack tip}

The ability of GBs in fcc metals to produce asymmetric crack growth was first found in an MD simulation of intergranular fracture in $\mathrm{Cu}$ by Cleri et al. (1999). In their work, a crack propagated in the interface plane of the symmetric tilt $(221) /(221)$ grain boundary. The crack advanced by brittle fracture along the [ $\left.\begin{array}{lll}1 & 1 & 4\end{array}\right]$ direction and was blunted by dislocation emission along the opposite $\left[\begin{array}{lll}\overline{1} & \overline{1} & 4\end{array}\right]$ direction. The difference in behavior at the two crack tips was attributed to the orientation of the slip planes relative to the GB. A slip plane inclined at angle $\theta$ to the GB makes the angle $\theta$ to the propagation direction of one crack tip, and the angle $\pi-\theta$ with the propagation direction of the opposing tip. Thus, for certain GBs, the Rice criterion (Rice, 1992) for dislocation nucleation versus cleavage might be satisfied for the crack tip at angle $\theta$, but not for the crack tip at an angle $\pi-\theta$, leading to asymmetric crack propagation. The 
To be submitted to: Journal of the Mechanics and Physics of Solids

crack grows primarily in the direction of the $\pi-\theta$ tip since the brittle propagation takes much less energy. Note that, for transgranular fracture, every direction in the fcc lattice has inverse symmetry, so such asymmetry in crack propagation is not expected to appear.

In the crystallographic model, presented in Fig. 2, the propagation direction $\left[\begin{array}{lll}\overline{7} & 7 & 10\end{array}\right]$ along the GB plane makes an angle $\theta=79.98^{\circ}$ (see Fig. 6(f)) with the twinning direction $\left[\begin{array}{lll}1 & 1 & 2\end{array}\right]$ in both crystals, giving $\cos \theta=1 / \sqrt{33}$. The Rice criterion for dislocation emission for the two propagation directions with $+\cos \theta(-x$ direction $)$ and $\cos (\pi-\theta)=-\cos \theta(+x$ direction $)$ can be written as

$$
\frac{\gamma_{u s}}{2 \gamma_{s}-\gamma_{G B}}<\frac{(1 \pm \cos \theta) \sin ^{2} \theta}{8}
$$

where the dislocations at the crack tip are edge twinning dislocations (shown as (3) in Fig. 6) with their Burgers vector coinciding with the twinning direction and lying in the $\left(\begin{array}{lll}1 & 1 & 0\end{array}\right)$ plane of the model. Using the values for $\gamma_{\mathrm{us}}, \gamma_{\mathrm{s}}$, and $\gamma_{\mathrm{GB}}$ given in Sec. 2.2, the left side of Eq. (4) equals $0.127 \pm 0.01$. The right side is determined to be 0.142 and 0.100 for the $-x$ and $+x$ propagation directions, respectively. Thus, the Rice criterion predicts that the crack tip should propagate in a ductile manner in the $-x$ direction, and in a brittle manner in the $+x$ direction, in agreement with the behavior seen in Fig. 5.

While the Rice criterion explains the ductile-brittle asymmetry of the crack found in the simulations (Figs. 6 and 7), an additional consideration is needed to explain the preference for twinning rather than the emission of slip dislocations in the $-x$ direction. Tadmor and Hai (2003) have recently derived a Pierels based criterion for the onset of deformation twinning at a crack tip in fcc metals by comparing the energies for nucleating a twinning or slip dislocation. This criterion introduces a so-called unstable twinning energy $\gamma_{\mathrm{ut}}$, which, by analogy with the unstable 
stacking-fault energy $\gamma_{\text {us }}$, gives the energetic barrier that must be exceeded to form a twin from an existing stacking-fault. The criterion is given by the parameter $T$ as

$$
T=\lambda \sqrt{\frac{\gamma_{u s}}{\gamma_{u t}}}=\left\{\begin{array}{cc}
<1 & \text { slip dislocation } \\
>1 & \text { deformation twin }
\end{array}\right.
$$

where $\lambda$ is a factor that is dependent on, both, the $\gamma_{\mathrm{sf}} / \gamma_{\mathrm{us}}$ ratio, and the orientation of the slip system of the twinning dislocations with respect to the crack plane (Tadmor and Hai, 2003). For the twinning tip in this simulation $\lambda=1.51$ (as applied from Tadmor and Hai, 2003), and

$\sqrt{\frac{\gamma_{u s}}{\gamma_{u t}}}=0.9$, which results in $T=1.36$. This suggests that twinning, rather than slip dislocation emission, will be the preferred deformation mechanism.

The brittle-ductile analysis based on the Rice criterion is for static or sufficiently slow crack growth. The criterion does not account for dynamic effects occurring at the crack tip propagating in the $+x$ direction (Yamakov et al., 2005) and cannot explain the observed dislocation burst in Fig. 7(f) (discussed in Section 3.2). Crack propagation is expected to be brittle throughout. Nevertheless, apart from these dynamic effects, there is good agreement between the simulation results and the two conditions expressed through Eqs. (4) and (5). This allows the prediction of the type of decohesion by knowing the crystallographic orientations of the grains and a few material parameters, such as the surface, GB, stacking-fault, and twinning energies.

\section{Plastic contribution to the stress field near the crack: MD - FE comparison}

The plastic processes at the crack tips, including twinning and dislocation emission, have a pronounced effect on the stress distribution near the growing crack. This effect is best revealed by a comparison between the stress distributions obtained from the MD and the linear elastic FE 
To be submitted to: Journal of the Mechanics and Physics of Solids

simulations for the models presented in Section 2. While the MD simulation considers the material structure at the atomic level and intrinsically incorporates all the plastic processes together with the elastic response of the bicrystal, the FE model assumes an elastically equivalent system and gives only the elastic solution. Another aspect of the FE simulation is that it gives a static solution when the system is in elastic equilibrium. By contrast, the MD simulation describes a continuously evolving system with all dynamic effects present. To avoid the implications of the dynamic effects in the comparison between FE and MD results, the corresponding MD stress distribution is calculated after the atomistic system reaches elastoplastic equilibrium and the crack stops growing. The dynamic solution for the elastic stress as a function of the crack velocity in the steady-state analytical model (Barber et al., 1989) is given by Ching (1994). Ching's solution is not studied here because extracting the non-equilibrium dynamic stress from MD is not a trivial task and goes beyond the scope of this work.

To obtain the continuum stress distribution from the MD simulation at elasto-plastic equilibrium, the virial formulation for the local stress is used, defined as (Cormier et al., 2001)

$$
\sigma_{\alpha \beta}=\frac{1}{\Omega} \sum_{i \in \Omega}\left(m^{(i)} v_{\alpha}^{(i)} v_{\beta}^{(i)}-\frac{1}{2} \sum_{j} \frac{\partial U}{\partial r^{(i, j)}} \frac{r_{\alpha}^{(i, j)} r_{\beta}^{(i, j)}}{\left|r^{(i, j)}\right|}\right)
$$

where $m^{(i)}$ and $v^{(i)}$ are the mass and velocity of the $i$-th atom, respectively, and $r^{(i, j)}=r^{(i)}-r^{(j)}$ is the vector between the positions of atoms $(i)$ and $(j)$ with $\alpha, \beta=1,2,3$ for the $x, y$, and $z$ Cartesian vector components. The first sum is taken over atoms $(i)$ in a volume $\Omega$ over which the stress is calculated, and the second sum is taken over atoms $(j)$, which are in the interaction range of the atom $(i)$. The derivative of the interaction potential $\frac{\partial U}{\partial r^{(i, j)}}$ is a generalization of the pair interatomic force between atoms $(i)$ and $(j)$ in the case of a many body potential. The volume $\Omega$ in this study is of size $6 a_{\mathrm{o}} \times 6 a_{\mathrm{o}} \mathrm{x} h$ in the $x$-, $y$-, and $z$-directions, respectively, which defines 
To be submitted to: Journal of the Mechanics and Physics of Solids

$\Omega=16.89 \mathrm{~nm}^{3}$ containing 864 atoms. This volume is large enough to ensure a good convergence of the calculated stress, but small enough to allow for a sufficiently high resolution of the stress distribution map. Additional time averaging of the stress is performed over 16 ps of simulation time, which is sufficient to smooth out the noise from the phonon waves in the simulation.

The virial theorem, which is the basis for calculating the local virial stress (Eq. (6)), provides the oldest and most frequently used expression for relating forces and motion within an atomic system to a continuum stress. The virial stress is defined through the local momentum flux carried by the particles in a small volume element (Lutsko, 1988), rather than as a force acting over a small surface element, i.e., the definition of the Cauchy stress used in continuum mechanics. Because the Cauchy stress assumes continuous structure of matter, the two definitions increasingly diverge at the atomic scale. However, in the limit of time and volume averages at equilibrium, the virial stress coincides with the Cauchy stress (Zimmerman et al., 2002).

The comparison between the stress distributions obtained from the FE and MD simulations for two cases of cracks are given in Figs. 8 and 9 in the form of two-dimensional $(x-y)$ stressmaps of the system created for each of the three stress components acting in the $(x-y)$ plane: $\sigma_{x x}$, $\sigma_{y y}$, and $\sigma_{x y}$. Fig. 8 presents the case of the small prestress of $3.5 \mathrm{GPa}$, which produces a $10 \mathrm{~nm}$ crack at equilibrium in the MD simulation (seen in Fig. 5(a)). The size of the crack is sufficiently small compared to the system size, and the elastic stress field calculated by the FE simulation (Figs. 8(a-c)) does not experience edge effects from the boundary conditions and thus resembles an infinite plate. The stress distribution for the brittle crack tip (the circled area in Figs. 8(d-f)) obtained by the MD simulation shows very good quantitative similarity to the FE result. This similarity suggests that the crack propagation in the MD system in the $+x$ direction is indeed 
To be submitted to: Journal of the Mechanics and Physics of Solids

brittle with essentially no plasticity. However, for the twinning tip there is a significant difference between the MD and FE results. The FE stress distribution is symmetric for the two crack tips, as they are elastically equivalent in the FE model (Figs. 8(a-c)). In contrast, twinning occurs in the MD system at the tip propagating in the $-x$ direction and significantly alters the stress field. The $\sigma_{x x}$ and $\sigma_{x y}$ stress components are largely relieved, while the $\sigma_{y y}$ stress is distributed along the twin boundaries (2) in Fig. 8(e)) and relieved at the crack tip. This reduction in local stress state explains the very slow crack propagation in the $-x$ direction of the MD model.

The GB layer, present in the MD model, but not in the FE model, increases the $\sigma_{x x}$ stress (1) in Figs. 8(d) and 9(d) corresponding to (1) in Figs. 5(a,b)), and suggests a stiffer GB layer. This stiffening effect should be taken with caution as the virial stress calculation in the MD simulation (Eq. (6)), as discussed previously, may give erroneous results when computed over small disordered atomic domains containing structural defects (e.g., GBs and surfaces, dislocation cores, etc., Zimmerman et al., 2002). It has been suggested that the highly constrained thermodynamic state of GBs in multilayer systems may appear stiffer than the crystal lattice in certain directions (Wolf and Jaszczak, 1993).

At a larger prestress of $3.75 \mathrm{GPa}$ the crack in the MD model reached almost $40 \mathrm{~nm}$ in length. The stress fields, presented in Fig. 9 (corresponding to the MD snapshot in Fig. 5(b)), are strongly affected by the system boundaries at $y= \pm W / 2$, as compared with the stress fields in Fig. 8. In the MD case (see Figs. 9(d-f) related to the snapshot in Fig. 5(b)), the stress field away from the crack tips is similar to the corresponding FE stress field, while the near-tip stress distribution is strongly affected by the plastic mechanisms at the crack tips. The contribution to the stress from the developed twinning on the $-x$ side of the crack (2) in Figs. 5(b) and 9(e)) and the few 
To be submitted to: Journal of the Mechanics and Physics of Solids

dislocations remaining in equilibrium with the crack tip on the $+x$ side (5) in Figs. 9(d-f)) can be easily identified. Both processes effectively relieve the stress around the crack tips as compared to the FE elastic solution (Figs. 9(a-c)) and lead the MD system into elasto-plastic equilibrium.

The stress-field maps discussed in this section are the basis for continuum interpretation of the atomistic MD simulations. They show that the stress around a crack tip, computed in the MD model, can be related to a continuum stress field. This makes it possible to use the MD simulations of crack propagation to extract the cohesive zone law for a grain-scale continuum model.

\section{Defining a Traction-Displacement Relationship from Molecular-Dynamics}

Grain-scale simulations that use cohesive zone models to study fracture (such as those presented by Zavattieri et al. (2001; 2003), Iesulauro et al. (2002), and Wei and Anand (2004)) typically use heuristically derived relationships and input values to define the CZM. In such an approach, the values at the microstructural scale are based on macroscopic parameters such as fracture toughness. Thus, there is no physical substantiation for the quantities that are input to the analysis. Conversely, a cohesive formulation can be part of an effective physics-based approach if constitutive parameters are used that exploit the similitude between atomistic simulation and continuum finite element results. The model can be developed from results of the MD analyses allowing the physical insight of MD to be embedded in the more computationally efficient FE models.

The traction-displacement function of a CZM describes how the traction $\tau$, developed in the process zone near the crack, depends on the crack opening $\lambda$. In general, the tractiondisplacement function $\tau(\lambda)$ considers both normal and tangential crack surface components of the 
To be submitted to: Journal of the Mechanics and Physics of Solids

traction. In the case of a normal opening (mode I) crack under hydrostatic load, only the normal component of the traction is important. In the MD simulation, the tensile $\sigma_{y y}^{\mathrm{s}}$ component of the stress near the debonding GB interface can be used as an equivalent of the normal traction $\tau_{n}$. The superscript ' $s$ ' indicates the stress near the GB interface.

To obtain $\sigma_{y y}^{\mathrm{s}}$ from MD, the simulation box is divided into $2 N_{L}+1$ slices of thickness $\delta$ (Fig. $10)$, so that $\left(2 N_{L}+1\right) \delta=L$. At the beginning of the simulation, before the crack is nucleated, a three-dimensional rectangular volume element (called a Cohesive-Zone-Volume-Element, CZVE) is defined at the place where each slice crosses the GB plane at $y=0$ (illustrated by the dotted regions in Fig. 10). The CZVE has a length $\delta_{x}$ along $x$ and extends to $y= \pm \delta_{y}$ on both sides of the GB interface. Its thickness equals the system thickness $h$. When the propagating crack passes through a CZVE during the simulation and the CZVE starts to open, its shape deforms (see the dotted elements at the crack tips in Fig. 10). To be able to keep track of the CZVE volume, the atoms that have belonged initially to the CZVE are marked, i.e., their identification numbers are stored for each CZVE, and are further used to identify the CZVE. Eq. (6), with the " $i$ "-index going over the marked atoms of a CZVE, and $\Omega$ equal to the volume of the CZVE, is used to calculate $\sigma_{y y}^{s}\left(x_{p}, t\right)$ as a function of the position $x_{p}$ of the $p$-th CZVE along the GB interface at time $t$ during the simulation. Here, $p \in\left[0, N_{L}\right]$ for the crack tip propagating in the $+x$ direction, and $p \in\left[0,-N_{L}\right]$ for the crack tip propagating in the $-x$ direction. At the same instant $t$, the crack opening $\lambda\left(x_{p}, t\right)$ at the same position $x_{p}$, is also estimated. Thus, every estimate of $\sigma_{y y}^{s}\left(x_{p}, t\right)$ corresponds to one estimate for $\lambda\left(x_{p}, t\right)$, allowing the construction of the function $\sigma_{y y}^{s}\left(\lambda\left(x_{p}, t\right)\right)$ for each CZVE.

An example of estimating $\sigma_{y y}^{s}\left(x_{p}, t\right)$ and $\lambda\left(x_{p}, t\right)$ for the case of a crack growing for $t=123 \mathrm{ps}$ in a system prestressed at $4.25 \mathrm{GPa}$ is given in Fig. 11. The stress and opening profiles $\left(\sigma_{y y}^{s}(x)\right.$ 
To be submitted to: Journal of the Mechanics and Physics of Solids

and $\lambda(x)$ curves, respectively) are taken from a set of CZVEs placed in a line along the debonding GB. A fit to the continuum expression for the stress at a crack tip

$$
\sigma_{y y}^{s}(x)=\frac{K_{I}}{\sqrt{2 \pi\left(x-x_{o}\right)}}+\sigma_{o},
$$

is imposed on the stress profile ahead of the brittle tip, propagating in the $+x$ direction (see Fig. 11). The fit shows a $1 / \sqrt{ } r$ type dependence of the stress profile and $K_{I}$ is determined to be 0.36 $\mathrm{MPa} \cdot \mathrm{m}^{1 / 2}$. Such dependence is not recovered for the ductile tip, propagating in the $-x$ direction in Fig. 11, because the stress is continuously relieved by the twinning process.

The $\sigma_{y y}^{s}(x)$ and $\lambda(x)$ profiles in Fig. 11 are for a single instant of time of the crack propagation. If the whole simulation of the interface debonding is divided into $N_{t}$ equal intervals of time $t_{q}\left(q \in\left[0, N_{t}\right]\right)$, many such profiles can be taken of many CZVEs placed along the GB. When plotted in a $\sigma_{y y}^{s}$ vs. $\lambda$ plot, each $\left(\sigma_{y y}^{s}\left(x_{p}, t_{q}\right), \lambda\left(x_{p}, t_{q}\right)\right)$ couple represents a point $\sigma_{y y}^{s}\left(\lambda\left(x_{p}, t_{q}\right)\right)$. After sorting these data points in an ascending order on $\lambda$, so that $\lambda_{i}<\lambda_{i+1}$, and taking a moving average (or a consecutive mean):

$$
\tau\left(\lambda_{i}\right)=\frac{1}{(2 M+1)} \sum_{j=-M}^{M} \sigma_{y y}^{s}\left(\lambda_{i+j}\right)
$$

in which the results are averaged over $M$ points backward and $M$ points forward from $\lambda_{i}$, a construction of a statistically representative traction-displacement function $\tau(\lambda)$ can be made.

The opening of the crack $\lambda(x, t)$ as a function of $x$ can be estimated in various ways. A convenient method is to use the previously described slicing of the simulation system along the $x$-axis (Fig. 10). The separation between atoms on either side of the GB interface increases as the crack passes through a slice, creating an opening of size $\lambda$ in the middle of the slice (the dashed slice in Fig. 10 is given as an example). The opening changes the slice's gyration radius $R_{g}$ (or 
To be submitted to: Journal of the Mechanics and Physics of Solids

the angular momentum), and one can estimate the size of the opening by calculating $R_{g}$. Assuming a uniform mass distribution, the continuum expression of $R_{g}$ as a function of $\lambda$ is

$$
R_{g}^{2}=\frac{\int_{\lambda / 2}^{W / 2} y^{2} d y}{\int_{\lambda / 2}^{W / 2} d y}=\frac{1}{12}\left(W^{2}+W \lambda+\lambda^{2}\right)
$$

Alternatively, expressed through the atomic coordinates, the discrete formula for $R_{g}$ is:

$$
R_{g}^{2}=\frac{1}{N} \sum_{i=1}^{N}\left(y_{i}-y_{C}\right)^{2}=\frac{1}{N} \sum_{i=1}^{N} y_{i}^{2}-\left(\frac{1}{N} \sum_{i=1}^{N} y_{i}\right)^{2}
$$

where $y_{i}$ is the $y$-coordinate of atom $i$, and the sum is over all $N$ atoms in the slice. $y_{C}=\frac{1}{N} \sum_{i=1}^{N} y_{i}$ is the $y$-coordinate of the mass center of the slice. Calculating $R_{g}$ from Eq. (10) and substituting it in Eq. (9), the crack opening at the slice is the positive root of $\lambda$ from Eq. (9).

Though the introduced CZVE is used to extract the behavior for the CZM element in the FE model, it should not be considered an MD equivalent of a CZM element. While the CZM elements are strictly surface elements in a 3D finite element model, the CZVE encompasses a volume at the surfaces on either side of the crack, large enough to allow for the convergence in the stress estimate in (Eq. (6). Each CZVE along the GB interface in the MD simulation has its individual behavior, depending on the local structural irregularities at the atomic level, while in a FE simulation the CZM elements for one type of interface are all identical. The relation between the CZVE and the CZM is made through statistical averaging (Eq. (8)). The assumption is made that under the steady-state conditions of crack propagation, the statistical average over many CZVEs scanned at different instants of time will produce a statistically unique tractiondisplacement curve. Assuming a Gaussian distribution of $\sigma_{y y}^{s}(\lambda(x, t))$ from each individual measurement of each CZVE, the dispersion around the average traction-displacement curve will 
To be submitted to: Journal of the Mechanics and Physics of Solids

decrease proportionally to the square root increase of the number of atoms belonging to the CZVE. Thus, a larger volume of the CZVE would better average out the statistical fluctuations. In MD simulations, this volume is limited by the small size of the damage zone around the crack tip and by the large stress gradients inside it. This makes the dispersion large, and a sufficient number of measurements is required to produce a reliable traction-displacement function.

The size of the CZVE is defined by $\delta_{x}=\delta_{y}=\delta=1 / 3\left[\begin{array}{lll}7 & 7 & 10\end{array}\right] \mathrm{a}_{\mathrm{o}} \approx 1.9 \mathrm{~nm}$ (see Fig. 10) for this simulation, which gives a volume of $2 h \delta^{2}=20.7 \mathrm{~nm}^{3}$ containing 1245 atoms that contribute to the stress-displacement response of one CZVE. For the simulated system in Fig. $1,2 N_{L}+1=63$ CZVEs fit along the $120 \mathrm{~nm}$ GB interface. During the simulation, $\sigma_{y y}^{s}(\lambda)$ state for each CZVE is scanned every 4 ps for a period of 200 ps crack propagation time. This gives 3150 points for $\sigma_{y y}^{s}(\lambda)$ dependence from each simulation run. These points provide adequate statistics to extract the traction-displacement curve through suitable averaging. Because the two crack tips propagate in a very different manner, as discussed in Section 3, the statistical averaging (Eq. (8)) must be performed separately for these two directions.

The values of $\sigma_{y y}^{s}(\lambda)$ for the brittle tip prestressed at $4.25 \mathrm{GPa}$ are given in Fig. 12(a). At 52 nm of propagation (Fig. 5(d)), 25 CZVEs have experienced a complete transition from a fully closed to a fully opened state. The results from 1575 calculations (along $0 \leq x \leq L / 2$ ), presented as points in Fig. 12(a), gives sufficient statistics to extract a reliable $\sigma_{\text {yy }}^{\mathrm{s}}(\lambda)$ curve using Eq. (8). The averaging interval is set to $M=100$, which is empirically found to give a sufficiently smooth curve. The curve reproduces closely a bilinear type of constitutive relation for the CZM element and is similar to the one suggested by Camacho and Oritz (1996) for brittle materials. The bilinear types of CZM models were used in several recent FE simulations of brittle fracture during multi-axial dynamic loading of ceramic microstructures (Zavattieri et al., 2001; 2003). 
To be submitted to: Journal of the Mechanics and Physics of Solids

The traction-displacement curves for 3.75 and $4.00 \mathrm{GPa}$, obtained in the same way, also given in Fig. 12(a) (the data points, over which the traction-displacement curves are averaged are omitted for clarity), show that varying the prestress of the system in this range does not change the essential character of the averaged $\sigma_{y y}^{s}(\lambda)$ dependence. The peak stress $\sigma_{p}$ shows a slight systematic decrease with decreasing load, and is probably an effect of the propagation speed decrease but remains approximately $\sigma_{p} \approx 5.0 \mathrm{GPa}$ at $\lambda_{p} \approx 0.4 \mathrm{~nm}$. It should be noted that the value for $\lambda_{p}$ is taken relative to the initial state at an applied hydrostatic loading of 3.75 to 4.25 GPa. In reality, $\sigma_{y y}^{s}(\lambda)$ for $\lambda<\lambda_{p}$ should follow the elastic response of the GB before debonding. The full opening, or full debonding, of the interface happens at $\lambda_{\mathrm{o}} \approx 2.5 \mathrm{~nm}$, when $\sigma_{y y}^{s}$ becomes zero.

The corresponding data $\sigma_{y y}^{s}(\lambda)$ for the ductile tip, propagating in the $-x$ direction where twinning occurs, is presented in Fig. 12(b). Again, only the data points for 4.25 GPa prestress are given for clarity, while the averaged traction-displacement curves are shown for the three loads of 3.75, 4.0 and 4.25 GPa, as in Fig. 12(a). In contrast with the behavior in the $+x$ direction, a stronger influence of the prestress on the traction-displacement curves is observed for the $-x$ direction. Most pronounced is this influence for $\lambda_{0}$, which changes from $\lambda_{0}{ }^{(1)}=2.7 \mathrm{~nm}$ to $\lambda_{0}{ }^{(3)}=$ $4.3 \mathrm{~nm}$ as the prestress is increased from 3.75 to $4.25 \mathrm{GPa}$. The peak stress $\sigma_{p}$ shows again the slight systematic decrease with decreasing load, which was observed in Fig. 12(a), accompanied by a shift in $\lambda_{p}$.

The comparison between the extracted traction-displacement relationships for the ductile and the brittle crack tips is presented in Fig. 13. The case of $4.25 \mathrm{GPa}$ prestress is chosen because there, the crack has achieved its largest growth in both directions (Fig. 5(d)) and has produced the most data points for CZVE statistics. Figure 13 reveals how the different atomistic processes, 
To be submitted to: Journal of the Mechanics and Physics of Solids

taking place at the two crack tips and discussed in detail in Sec. 3, are affecting the decohesion law of the interface debonding. This comparison is also very informative on the significance of the form of the traction-displacement curve and how this form reflects the underlying physical processes as revealed by the presented atomistic MD simulations. The ductile tractiondisplacement curve is substantially more extended towards larger debonding distances $\lambda_{0}$, compared to the case of almost brittle debonding $\left(\lambda_{0}{ }^{d t}>\lambda_{0}{ }^{b r}\right.$, see Fig. 13). The corresponding peak stress $\sigma_{p}$ is also lower for the ductile case, which is possibly a result of the emission of twinning dislocations relieving the stress at the crack tip. The lower $\sigma_{p}$ and the larger $\lambda_{\mathrm{o}}$ during twinning produces a more extended debonding region for the ductile traction-displacement curve, compared to the steeper curve in the case of brittle fracture. This difference justifies the use of a trapezoidal type of curve with a plastic straining region and reduced peak stress for the case of elasto-plastic fracture (Tvergaard and Hutchinson, 1996) and a bilinear curve for brittle fracture (Camacho and Ortiz, 1996). Despite a lower peak stress, the traction-displacement curve with twinning has about 50\% larger area, meaning a larger work of separation $\Gamma$ defined as (Tvergaard and Hutchinson, 1992)

$$
\Gamma=\int_{0}^{\lambda_{0}} \sigma_{y y}^{s} d \lambda
$$

The larger work of separation also explains the smaller propagation distance in the ductile $(-x)$ direction, compared to the brittle $(+x)$ direction in the MD simulation (see Fig. 5(d) and compare also Figs. 6(a-f) with Figs. 7(a-f) discussed in Section 3).

\section{Conclusions}

A methodology is detailed for extracting the decohesion law for interface debonding by introducing a Cohesive-Zone-Volume-Element using molecular-dynamics simulations. The 
To be submitted to: Journal of the Mechanics and Physics of Solids

methodology is applied for the process of debonding of a high-energy $\sum 99 \mathrm{~GB}$ in Al through crack propagation under hydrostatic loading conditions. The work reveals the atomic mechanisms in the damage zone near the crack tips during intergranular fracture. Here, the crack propagation has been shown to proceed in a different manner in the two opposite directions along the GB interface. While in one direction the crack progresses in a brittle manner, the propagation in the opposite direction is of a ductile type, characterized by the presence of plastic mechanisms including twinning at the crack tip. The difference in the mechanism is due to the inclination of the slip planes to the GB interface making different angles with respect to the two opposite propagation directions.

Applying a statistical procedure, the decohesion laws in terms of traction-displacement curves for the brittle and ductile crack propagations are extracted from the atomic forces and motions around the crack tips. The comparison between these curves reveals the influence of the plastic mechanisms on the behavior of the CZM element. The appearance of plastic mechanisms extends the traction-displacement curve towards larger openings, while reducing the peak opening traction. This results in an increase of the area below the curve, as the work of decohesion increases in the presence of plastic processes. This justifies the use of an empirically derived trapezoidal type of decohesion law for the case of solids with enhanced plasticity and a bilinear law for britle materials.

The brittle vs. ductile propagation simulated in both directions along the GB interface is found to agree well with the predicting criteria of Rice and Tadmor-Hai. This good compliance with the two criteria allows the selection of the proper cohesive zone model in a finite element simulation by knowing the crystallographic orientations of the grains in the microstructure and a few material parameters. The parameters, including surface, GB, stacking-fault, and twinning 
To be submitted to: Journal of the Mechanics and Physics of Solids

energy, are readily available experimentally. Others, such as the unstable stacking-fault energy and the unstable twinning energy, need more precise estimates and may require the use of firstprincipal calculations.

\section{Acknowledgements}

V. Yamakov and D. R. Phillips were sponsored through cooperative agreement NCC-102043 with the National Institute of Aerospace and contract NAS1-00135 with Lockheed Martin Space Operations, respectively. 
To be submitted to: Journal of the Mechanics and Physics of Solids

\section{Appendix A}

Molecular-dynamics (MD) monocrystal simulations were used to estimate the stiffness coefficients of an aluminum single crystal at $100 \mathrm{~K}$ in the basic orientation: $\left(x:\left[\begin{array}{llll}1 & 0 & 0\end{array}\right] ; y:\left[\begin{array}{lll}0 & 1 & 0\end{array}\right]\right.$; $\left.z:\left[\begin{array}{lll}0 & 0 & 1\end{array}\right]\right)$. The values obtained are (in GPa): $\mathrm{C}_{11}=109.1 ; \mathrm{C}_{12}=58.5 ; \mathrm{C}_{44}=33.0$. While $\mathrm{C}_{11}$ and $\mathrm{C}_{12}$ are slightly lower than the zero temperature value for the potential used (Mishin et al., 1999), $\mathrm{C}_{44}$ is slightly higher. This increases slightly the anisotropy parameter $\mathrm{C}_{\mathrm{A}}=2 \mathrm{C}_{44} /\left(\mathrm{C}_{11}-\mathrm{C}_{12}\right)=1.30$ compared to the zero temperature $\mathrm{C}_{\mathrm{A}}(\mathrm{T}=0 \mathrm{~K})=1.21$. The transformation of the stiffness coefficients to the $\sum 99$ crystal orientations, given in Section 2.2, is performed according to the shortened for cubic crystals procedure (Wortman and Evans, 1965), which gives the following stiffness matrix

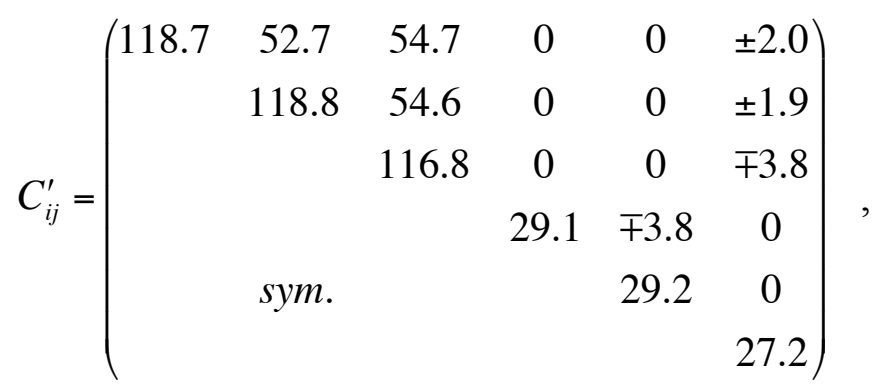

where the prime indicates transformed elastic constants with $\mathrm{C}_{i j}^{\prime}=\mathrm{C}_{j i}^{\prime}$. The " \pm " sign at some of the off-diagonal values indicate change of sign when going from Crystal I orientation to Crystal II orientation. Due to the small anisotropy, the stiffness matrix in Eq. (A.1) almost preserves its cubic symmetry with $\mathrm{C}_{11}^{\prime} \approx \mathrm{C}_{22}^{\prime} \approx \mathrm{C}_{33}^{\prime} \approx 118, \mathrm{C}_{12}^{\prime} \approx \mathrm{C}_{13}^{\prime} \approx \mathrm{C}_{23}^{\prime} \approx 54, \mathrm{C}_{44}^{\prime} \approx \mathrm{C}_{55}^{\prime} \approx \mathrm{C}_{66}^{\prime} \approx 29$, and the remaining $\mathrm{C}_{i j}^{\prime} \approx 0$. Using these approximate values in the $\mathrm{FE}$ model makes the two crystallographic orientations on both sides of the GBs elastically equivalent. This significantly simplifies the problem from a slit in a multilayer system to a slit in a single cubic crystal with the GBs neglected (see Fig. 3). 
To be submitted to: Journal of the Mechanics and Physics of Solids

\section{References:}

ABAQUS/Standard User's Manual, Hibbitt, Karlsson, and Sorensen, Inc., 2004.

Abraham, F.F., 2001.The atomic dynamics of fracture. J. Mech. Phys. Solids 49, 2095-2111.

Abraham, F.F., Brodbeck, D., Rafey, R.A., Rudge, W.E., 1994. Instability dynamics of fracture: A computer simulation investigation. Phys. Rev. Lett. 73, 272-275.

Allen, M. P., Tildesley, J. P., 1989. Computer simulations of liquids (Clarendon Press, New York, NY, USA), p.385.

Barber, M., Donley, J., Langer, J. S., 1989. Steady-state propagation of a crack in a viscoelastic strip. Phys. Rev. A 40, 366-376.

Camacho, G.T., Ortiz, M., 1996. Computational modeling of impact damage in brittle materials. Int. J. Solids Struct. 33, 2899-2938.

Chen, Q., Huamg, Y., Quiao, L., Chu, W., 1999. Failure modes after exhaustion of dislocation glide ability in thin crystals. Sci. China (Series E) 42, 1-9.

Ching, E. S. C., 1994. Dynamic stresses at a moving crack tip in a model of fracture propagation. Phys. Rev. E 49, 3382-3388.

Clarke, A. S., Jonsson, H., 1993. Structural changes accompanying densification of random hard-sphere packings. Phys. Rev. E 47, 3975-3984.

Cleri, F., Phillpot, S.R., Wolf, D., 1999. Atomistic simulations of intergranular fracture in symmetric-tilt grain boundaries. Interface Sci. 7, 45-55.

Cormier, J., Rickman, J. M., Delph, T. J., 2001. Stress calculation in atomistic simulations of perfect and imperfect solids. J. Appl. Phys. 89, 99-104.

Costanzo, F., Allen, D.H., 1995. A continuum thermodynamic analysis of cohesive zone models. International J. Sci. Eng. 33, 2197-2219.

Dahmen, U., Hetherington, J.D., O’Keefe, M.A., Westmacott, K.H., Mills, M.J., Daw, M.S., Vitek, V., 1990. Atomic Structure of a $\Sigma 99$ Grain Boundary in Al: a Comparison Between Atomic- 
To be submitted to: Journal of the Mechanics and Physics of Solids

Resolution Observation and Pair-Potential and Embedded-Atom Simulations, Philos. Mag. Lett. $62,327-335$.

Dávila, C. G., 2001. Mixed-mode decohesion elements for analysis of progressive delamination. $42^{\text {nd }}$ AIAA/ASME/ASCE/AHS/ASC Structures, Structural Dynamics, and Materials Conference and Exhibit, Seattle, WA April, 16-19 2001, article: AIAA-01-1486.

Farkas, D., 2000a. Bulk and intergranular fracture behavior of NiAl. Philos. Mag. A 80, 1425-1444.

Farkas, D., 2000b. Fracture mechanisms of symmetrical tilt grain boundaries. Philos. Mag. Lett. 80, 229-237.

Farkas, D., Duranduru, M., Curtin, W.A., Ribens, C., 2001. Multiple-dislocation emission from the crack tip in the ductile fracture of Al. Philos. Mag. A 81, 1241-1255.

Gall, K., Horstemeyer, M.F., Van Schilfgaarde, M., Baskes, M.I., 2000. Atomistic simulations on the tensile debonding of an aluminum-silicon interface. J. Mech. Phys. Solids 48, 2183-2212.

Gao, H., 1996. A theory of local limiting speed in dynamic fracture. J. Mech. Phys. Solids 44, 14531474.

Gumbsch, P., Zhou, S. J., Holian, B. L., 1997. Molecular dynamics investigation of dynamic crack stability. Phys. Rev. B 55, 3445-3455.

Hai, S., Tadmor, E.B., 2003. Deformation twinning at aluminum crack tips. Acta Mater. 51, 117-131.

Honeycutt, J. D., Andersen, H. C., 1987. Molecular dynamics study of melting and freezing of small Lennard-Jones clusters. J. Phys. Chem. 91, 4950-4963.

Iesulauro, E., Ingraffea, A. R., Arwade, S., Wawrzynek, P. A., 2002. Simulation of grain boundary decohesion and crack initiation in aluminum microstructure models, in Fatigue and Fracture Mechanics: $33^{\text {rd }}$ Volume, ASTM STP 1417, (W. G. Reuter and R. S. Piascik, Eds., American Society for Testing and Materials, West Conshohocken, PA, 2002).

Klein, P., Gao, H., 1998. Crack Nucleation and Growth as Strain Localization in a Virtual-Bond Continuum. Eng. Fract. Mech. 61, 21-48.

Komanduri, R., Chandrasekaran, N., Raff, L.M., Molecular dynamics (MD) simulation of uniaxial tension of some single-crystal cubic metals at nanolevel. Int. J. Mech. Sci. 43, 2237-2260. 
To be submitted to: Journal of the Mechanics and Physics of Solids

Langer, J. S., 1992. Models of crack propagation. Phys. Rev. A 46, 3123-3131.

Langer, J. S., 1993. Dynamic model of onset and propagation of fracture. Phys. Rev. Lett. 70, 35923594.

Langer, J. S., Nakanishi, H., 1993. Models of crack propagation. II. Two-dimensional model with dissipation on the fracture surface. Phys. Rev. E 48, 439-448.

Lutsko, J. F., 1988. Stress and elastic constants in anisotropic solids: molecular dynamics techniques. J. Appl. Phys. 64, 1152-1154.

Mishin, Y., Farkas, D., Mehl, M.J., Papaconstantopoulos, D.A., 1999. Interatomic potentials for monoatomic metals from experimental data and ab initio calculations. Phys. Rev. B 59, 33933407.

Nose, S., 1984. A Unified Formulation of the Constant Temperature Molecular Dynamics Method. J. Chem. Phys. 81, 511-519.

Parrinello, M., Rahman, A., 1981. Polymorphic Transitions in Single Crystals; A New Molecular Dynamics Method. J. Appl. Phys. 52, 7182-7190.

Pond, R.C., Garcia-Garcia, L.M.F., 1982. Deformation twinning in Al. Inst. Phys. Conf. Series 61, 495498.

Raynolds, J.E., Smith, J.R., Zhao, G.-L., Srolovitz, D.J., 1996. Adhesion in NiAl-Cr from first principles. Phys. Rev. B 53, 13883-13890.

Rice, J. R., 1992. Dislocation nucleation from a crack tip: an analysis based on the Peierls concept. J. Mech. Phys. Solids 40, 239-271.

Schiotz, J., Vegge, T., Di Tolla, F. D., Jacobsen, K. W., 1999. Atomic-scale simulations of the mechanical deformation of nanocrystalline metals. Phys. Rev. B 60, 11971-11983.

Spearot, D., Jacob, K.I., McDowell, D.L., 2004. Non-local separation constitutive laws for interfaces and their relation to nanoscale simulations. Mech. Mater. 36 (2004) 825-847

Tadmor, E.B., Hai, S., 2003. A Peierls criterion for the onset of deformation twinning at a crack tip. J. Mech. Phys. Solids 51, 765-793. 
To be submitted to: Journal of the Mechanics and Physics of Solids

Tvergaard, V., Hutchinson, J.W., 1992. The Relation Between Crack Growth Resistance and Fracture Process Parameters in Elastic-Plastic Solids. J. Mech. Phys. Solids 40, 1377-1397.

Tvergaard, V., Hutchinson, J.W., 1996. Effectt of strain-dependent cohesive zone model on predictions of crack growth resistance. Int. J. Solids Struct. 33, 3297-3308.

Weertman, J., Weertman, J. R., 1992. Elemantary dislocation theory. (Oxford University Press, New York, 1992).

Wei, Y. J., L. Anand, L., 2004. Grain-boundary sliding and separation in polycrystalline metals: application to nanocrystalline fcc metals. J. Mech. Phys. Solids 52, 2587-2616.

Wolf, D., 1990. Correlation between structure, energy, and ideal cleavage fracture for symmetrical grain boundaries in fcc metals. J. Mater. Res. 5, 1708-1730.

Wolf, D., Jaszczak, J. A., 1993. Tailored elastic behavior of multilayers through controlled interface structure. J. Comp. Aided Mater. Design 1, 111-148.

Wortman, J. J., Evans, R. A., 1965. Young's modulus, and Poisson's ratio in silicon and germanium. J. Appl. Phys. 36, 153-156.

Yamakov, V., Saether, E., Phillips, D. R., Glaessgen, E. H., 2005. Dynamic Instability in Intergranular Fracture. Phys. Rev. Lett. 95, 015502-1-4.

Yamakov, V., Wolf, D., Phillpot, S.R., Mukherjee, A.K, Gleiter, H., 2002. Dislocation Processes in the Deformation of Nanocrystalline Al by Molecular-Dynamics Simulation. Nature Mater. 1, 45-48. Yamakov, V., Wolf, D., Phillpot, S.R., Gleiter, H., 2003a. Dislocation-Dislocation and DislocationTwin Reactions in Nanocrystalline Al by Molecular-Dynamics Simulation. Acta Mater. 51, 41354147.

Yamakov, V, Wolf, D, Phillpot, S. R, Mukherjee, A. K, Gleiter, H., 2003b. Deformation mechanism crossover and mechanical behavior in nanocrystalline materials. Philos. Mag. Lett. 83, 385-393.

Yamakov, V., Wolf, D., Salazar, M., Phillpot, S.R., and Gleiter, H., 2001. Length-Scale Effects in the Nucleation of Extended Lattice Dislocations in Nanocrystalline Al by Molecular-Dynamics Simulation. Acta Mater. 49, 2713-2722. 
To be submitted to: Journal of the Mechanics and Physics of Solids

Zavattieri, P.D., Raghuram, P.V., Espinosa, H.D., 2001. A computational model of ceramic microstructures subjected to multi-axial dynamic loading. J. Mech. Phys. Solids 49, 27-68.

Zavattieri, P.D., Espinosa, H.D., 2003. An examination of the competition between bulk behavior and interfacial behavior of ceramics subjected to dynamic pressure-shear loading. J. Mech. Phys. Solids 51, 607-635.

Zimmerman, J.A., Jones, R.E., Klein, P.A., Bammann, D.J., Webb III, E.B., Hoyt, J.J., 2002. Continuum definitions for stress in atomistic simulation, SAND Report, Sandia National Laboratory, SAND2002-8608. 
To be submitted to: Journal of the Mechanics and Physics of Solids

\section{Figures:}

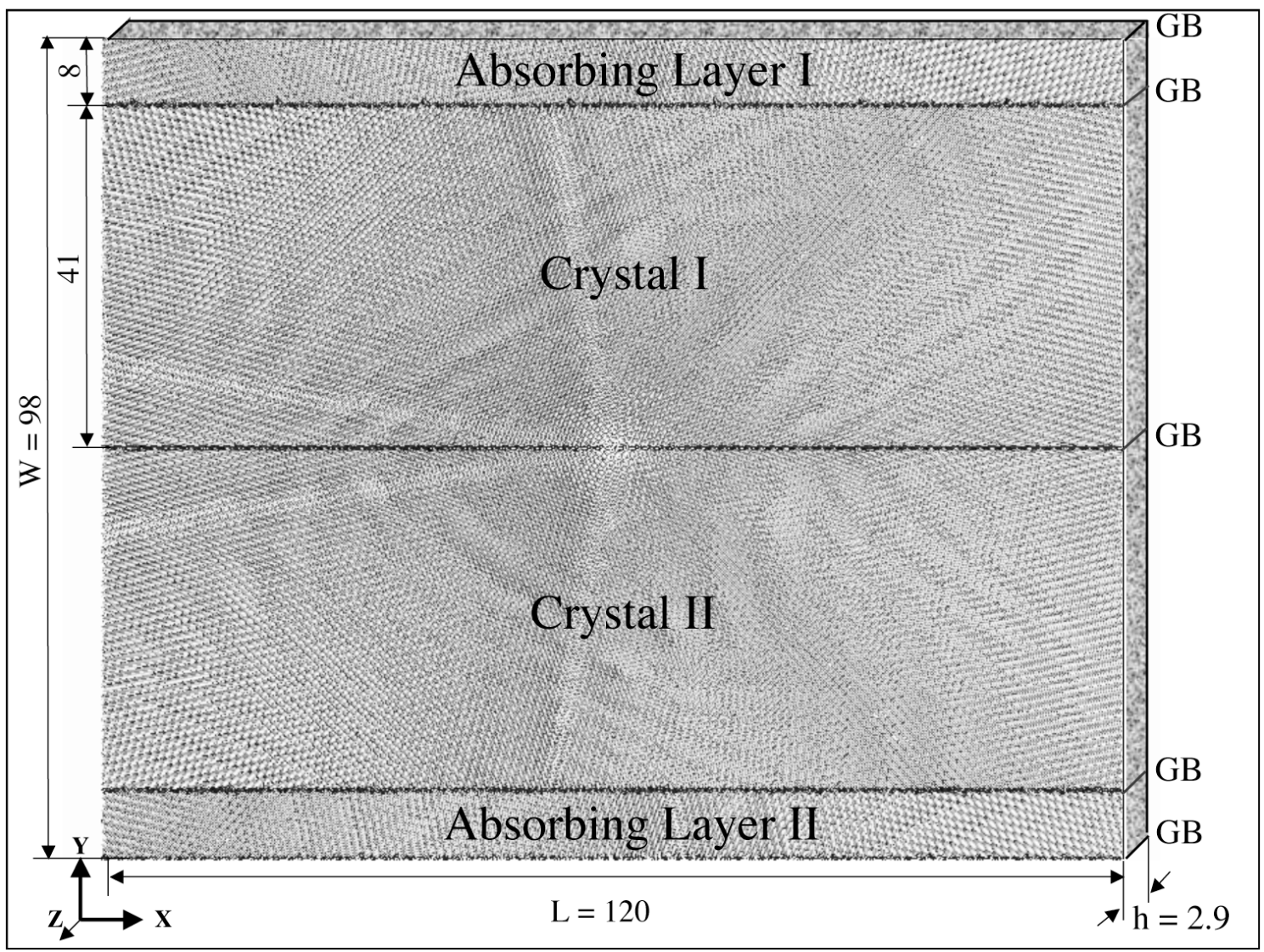

Fig. 1. Initial atomistic snapshot of the molecular-dynamics (MD) system before crack initiation, representing the simulation set-up, explained in the text. Size dimensions are in nm. Grain boundaries (GB) are shown as parallel lines of dark atoms separating the crystalline phases of Crystal I, Crystal II, and Absorbing Layers I and II as indicated. 


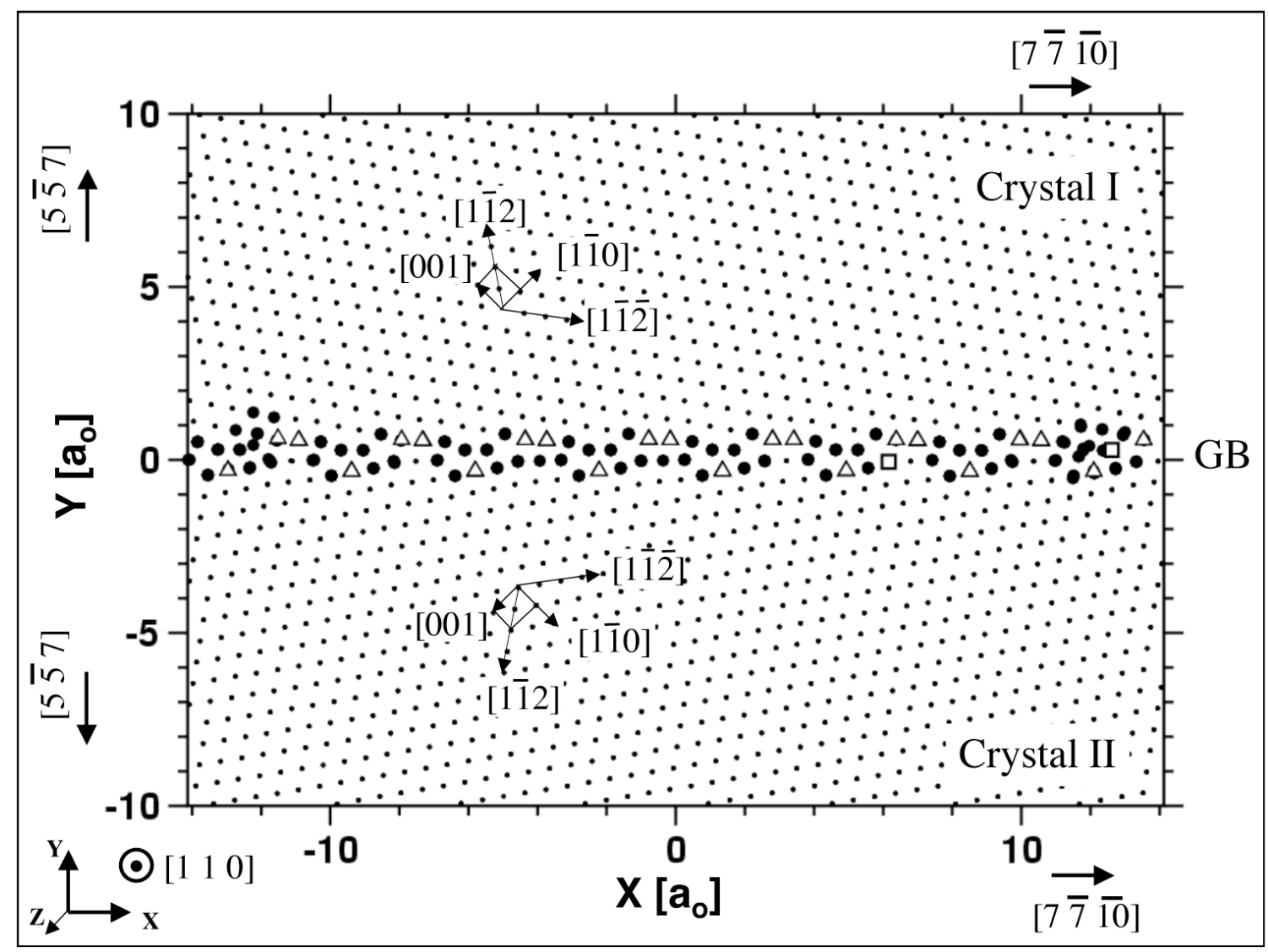

Fig. 2. Atomistic snapshot giving the crystallography and structure of the GB interface. Common neighbor analysis (CNA) (Honeycutt and Andersen, 1987; Clarke and Jonsson, 1993) is used to identify atoms in different crystallographic states: fcc (small dots), hep (triangles), and noncrystalline atoms (large dots). Atoms with more than 1/3 of their nearest neighbors missing are identified as surface atoms (squares), indicating existing vacancies in the GB. The length scale is in units of the lattice constant of $\mathrm{Al}, \mathrm{a}_{\mathrm{o}}=0.405 \mathrm{~nm}$ (Mishin et al., 1999). 
To be submitted to: Journal of the Mechanics and Physics of Solids

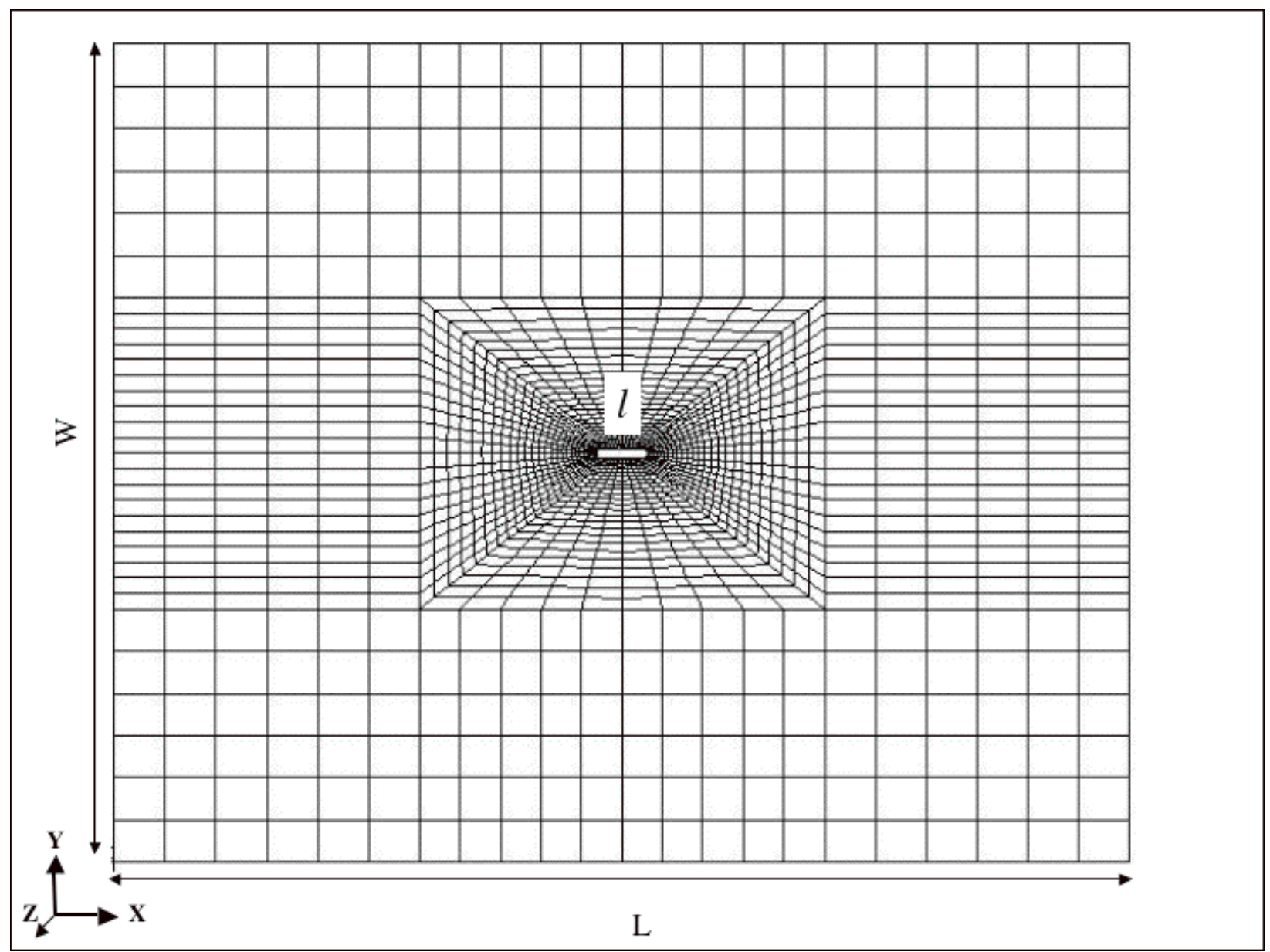

Fig. 3. The mesh of the finite element model with a built in lenticular crack. The relative crack length, $l / L$, varied from 0.05 to 0.91 , corresponding to the absolute crack length from 6 to 110 $\mathrm{nm}$ in the MD system, shown in Fig. 1. Generalized plane strain in the $z$-direction is used to emulate the hydrostatic loading conditions in the MD system, shown in Fig. 1. 
To be submitted to: Journal of the Mechanics and Physics of Solids

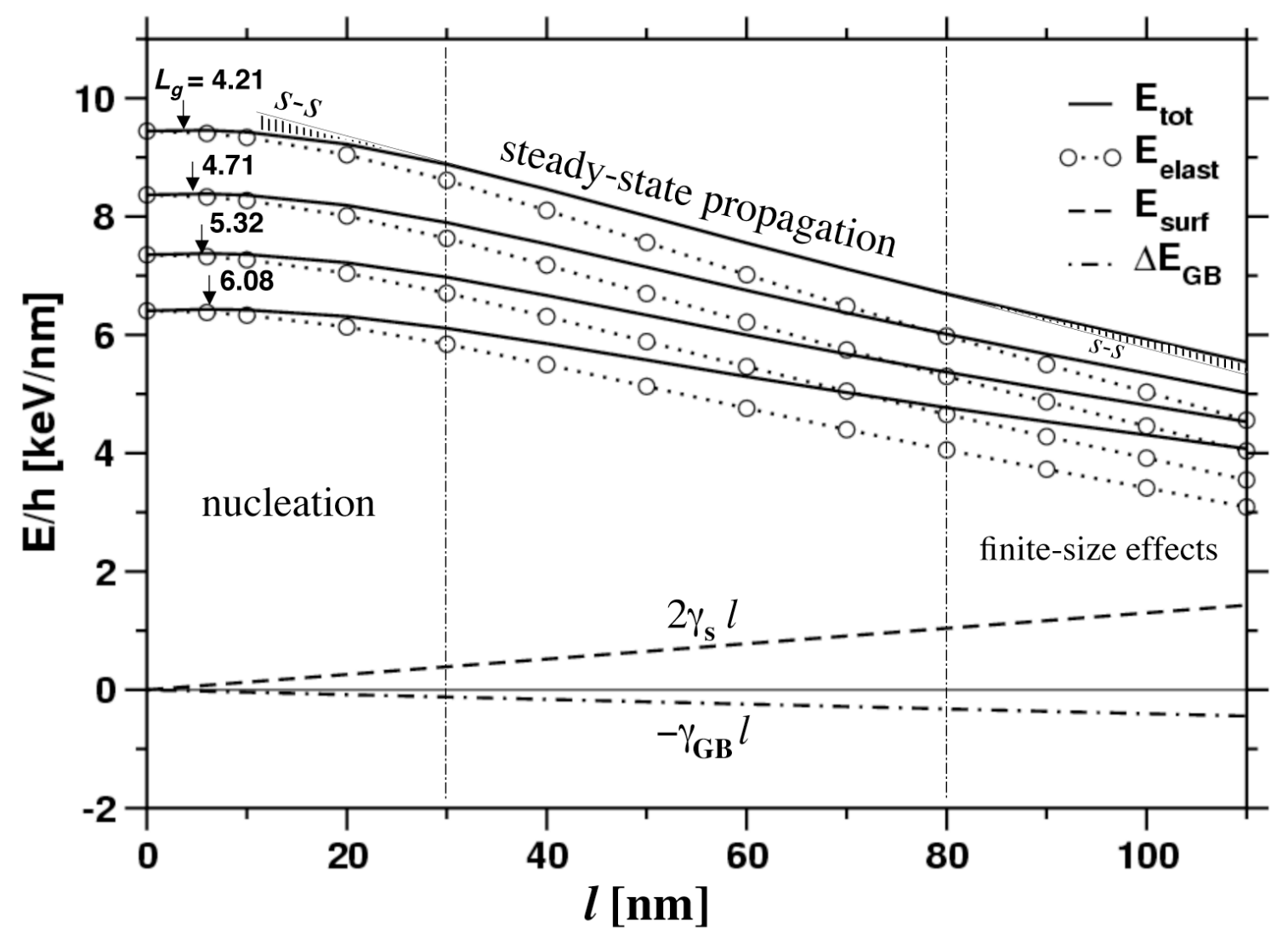

Fig. 4. The strain energy per unit thickness, $E / h$, of the FE system with increasing crack length $l$. The two straight, dashed, and dot-dashed lines, $E_{\text {surf }}=2 \gamma_{\mathrm{s}} l$ and $\Delta E_{G B}=-\gamma_{G B} l$, show the effective increase of the surface energy and the decrease of the GB energy with increasing $l$. The position of the maximum of $E_{\text {tot }}=E_{\text {elast }}+E_{\text {surf }}+\Delta E_{G B}$ indicates the Griffith length $L_{g}$, as shown for the four applied prestresses of 4.25, 4.0, 3.75, and 3.5 GPa, starting from the top down. 
To be submitted to: Journal of the Mechanics and Physics of Solids
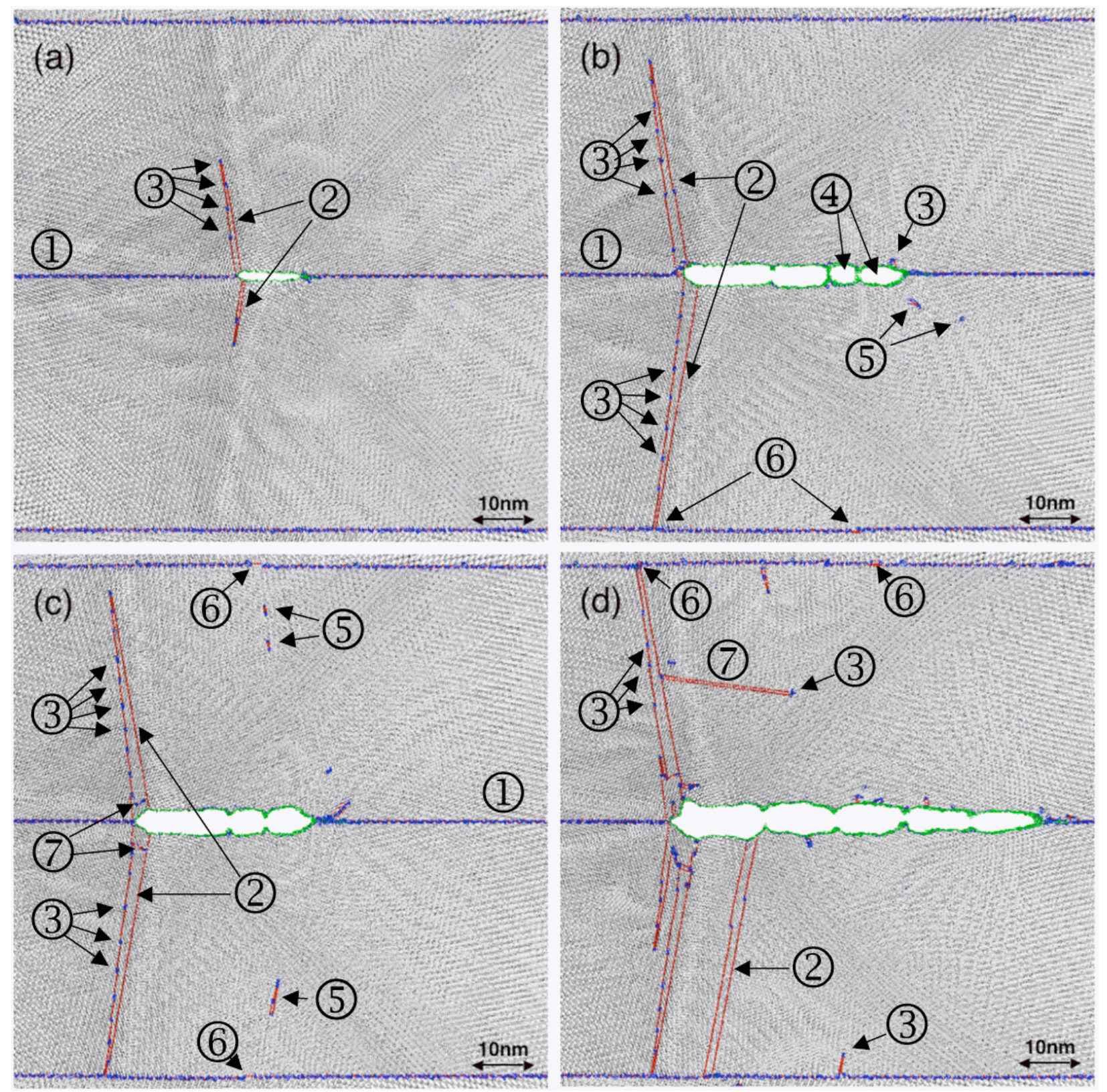

Fig. 5. (In color) MD snapshots of cracks, which have propagated in the MD system, shown in Fig. 1, prestressed at four different initial hydrostatic stresses: $\sigma=3.5$ (a), 3.75 (b), 4.0 (c) and 4.25 GPa (d). As in Fig. 2, CNA is used to identify atoms in different crystallographic states: fcc (in gray), hcp (in red), and non-crystalline atoms (in blue). Atoms with more than 1/3 of their nearest neighbors missing are identified as surface atoms (in green). Thus, a number of different formations are indicated in the figure as follows: (1) - GB interface; (2) - twin boundary; (3) - core of a partial or twinning dislocation; (4) - nanovoid at the crack tip; (5) - slip dislocation; (6) - GB dislocation; and (7) - secondary slip. 
To be submitted to: Journal of the Mechanics and Physics of Solids
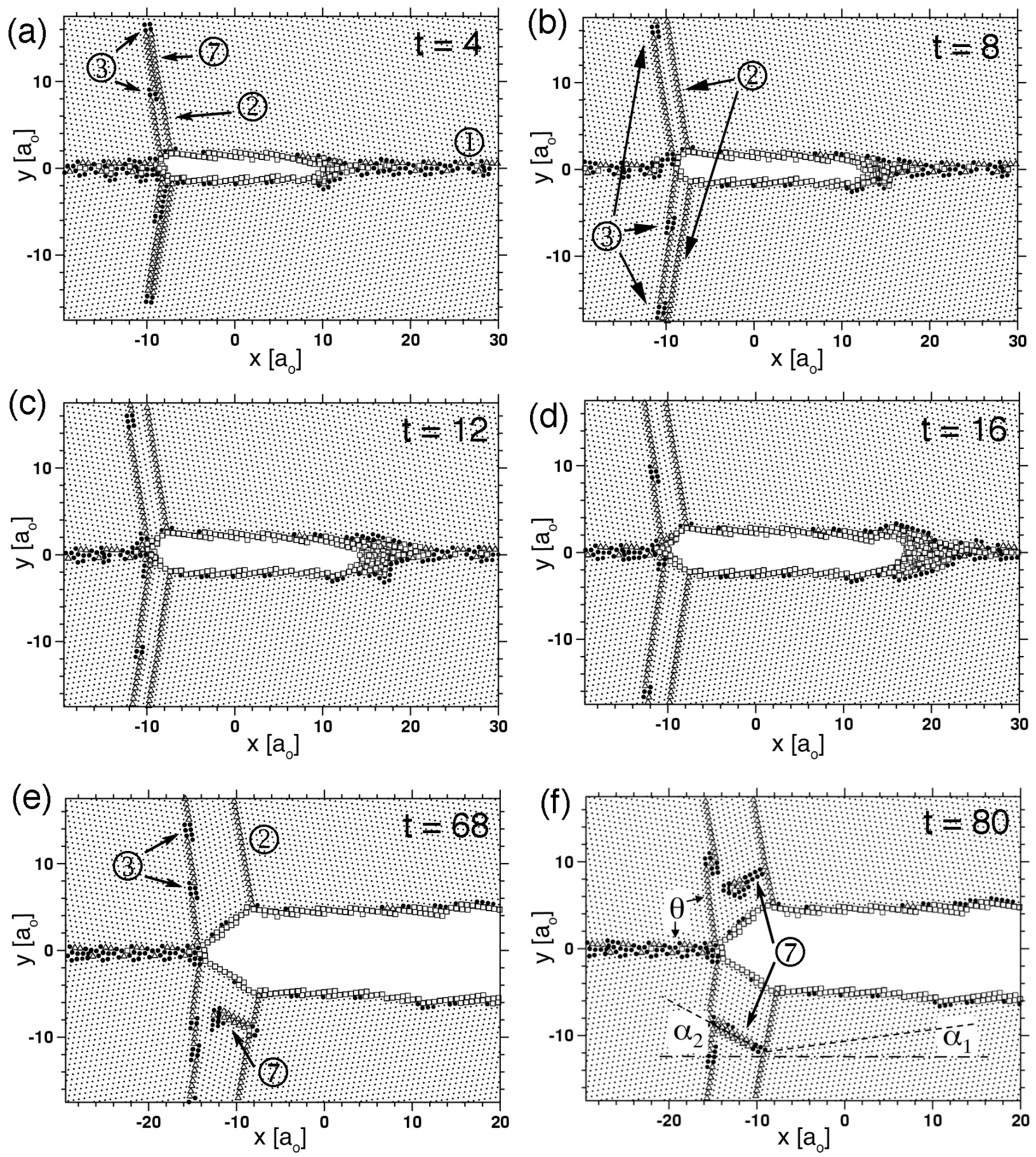

Fig. 6. A series of snapshots monitoring the development of a symmetrical deformation twinning at the $-x$ crack tip in the MD system prestressed at 4.0 GPa hydrostatic load. The snapshots are taken at various times from the crack initiation, $t$, given in ps. The length scale is in units of the lattice constant of $\mathrm{Al}, \mathrm{a}_{\mathrm{o}}=0.405 \mathrm{~nm}$. Identified as in Fig. 5, the small dots indicate fcc atoms; the non-crystalline atoms are shown in black, while hep and surface atoms are shown in grey. Formations (1), (2), (3), and (7) correspond to the types indicated in Fig. 5. 
To be submitted to: Journal of the Mechanics and Physics of Solids
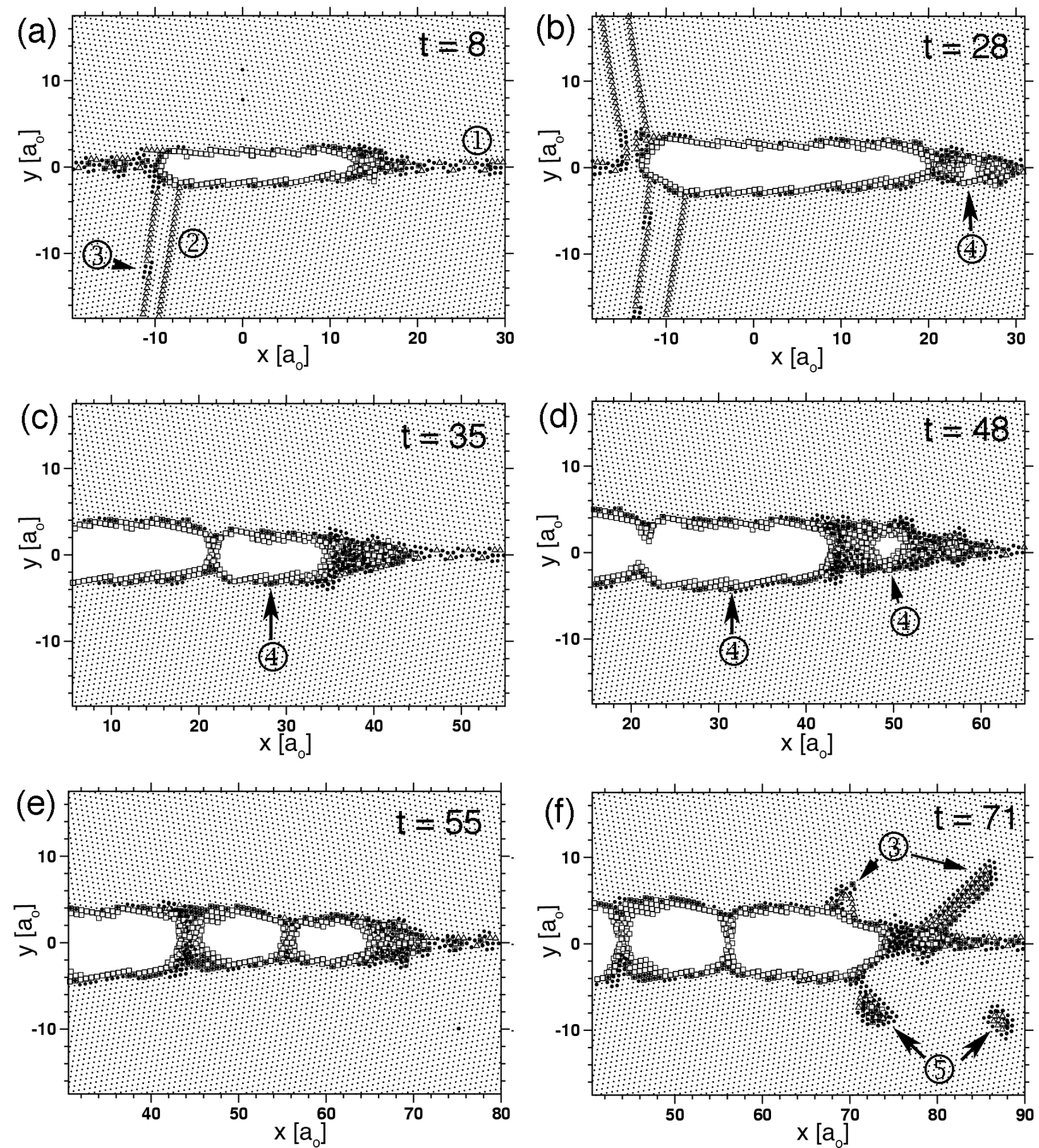

Fig. 7. A series of snapshots monitoring the propagation of the crack tip in the $+x$ direction in the MD system prestressed at $3.75 \mathrm{GPa}$ hydrostatic load. The snapshots are taken at various times from the crack initiation, $t$, given in ps. The length scale is in units of the lattice constant of $\mathrm{Al}, \mathrm{a}_{\mathrm{o}}$ $=0.405 \mathrm{~nm}$. The atoms of different types are identified as in Fig. 6. Formations (1)-(5) correspond to the types indicated in Fig. 5. 
To be submitted to: Journal of the Mechanics and Physics of Solids
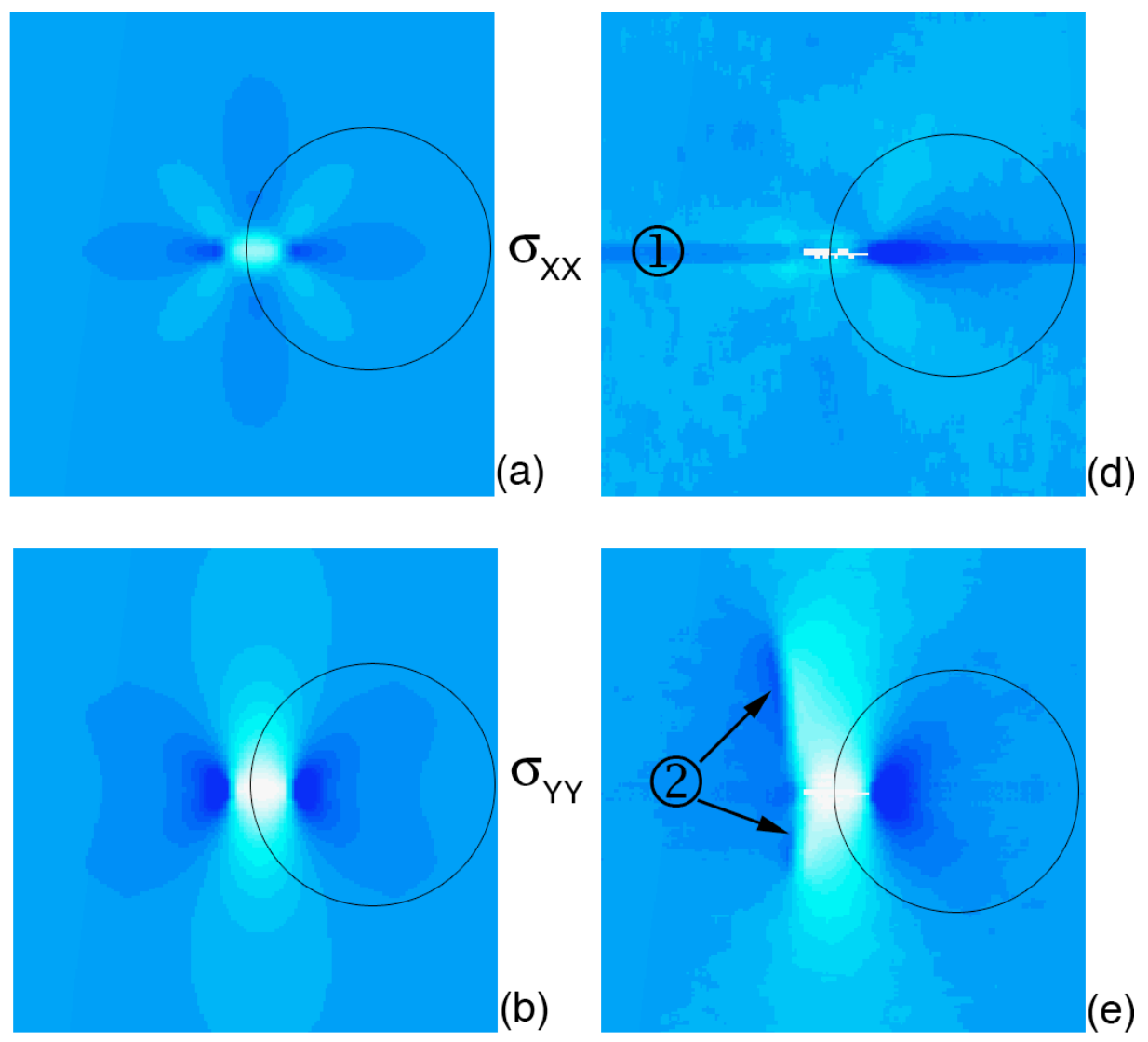

0

$4.8 \mathrm{GPa}$

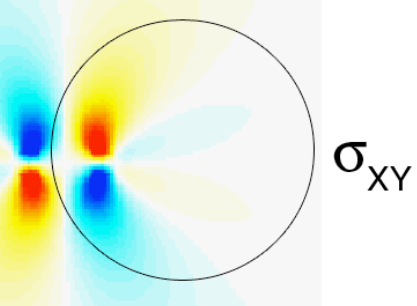

(c)

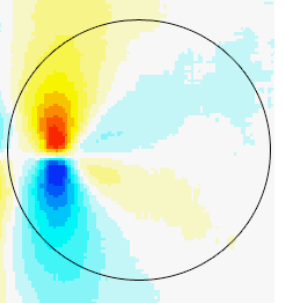

(f)

Fig. 8. (In color) Stress contours from the FEM model (a-c) (corresponding to Fig. 3) and the corresponding stress maps (d-f) from the MD model (corresponding to Fig. 5(a) at $3.5 \mathrm{GPa}$ prestress) for $\sigma_{x x}, \sigma_{y y}$, and $\sigma_{x y}$ stress components. In (a), (b), (d), and (e), the stress in blue is defined as the stress in tension. In (c) and (f), positive and negative shear corresponds to shear directions relative to the GB interface as shown at the two sides of the $\sigma_{x y}$ stress indicator. Formations (1) and (2) correspond to those indicated in Fig. 5(a). 
To be submitted to: Journal of the Mechanics and Physics of Solids
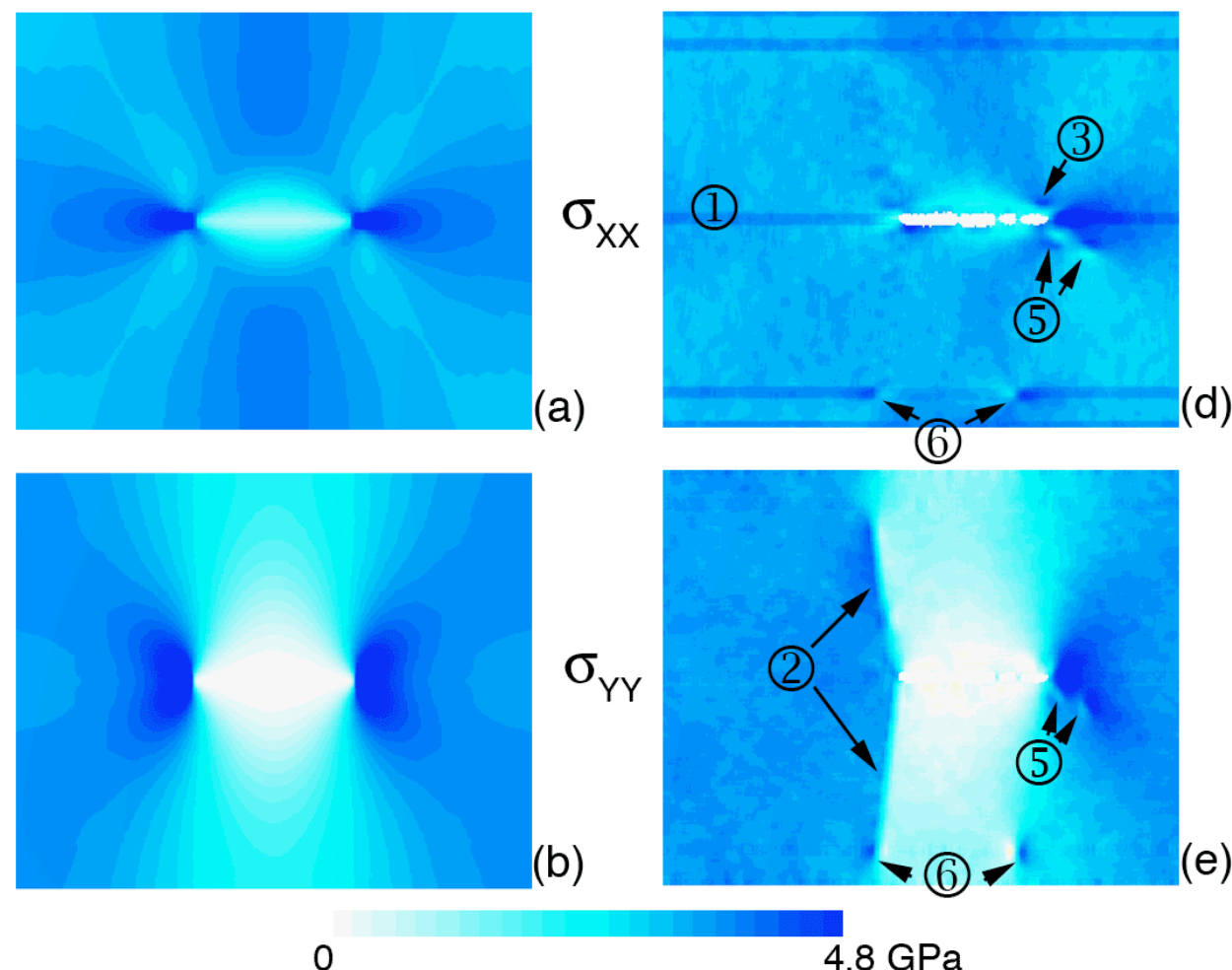

\section{$\sigma_{X Y}$}

(c)

(f)

Fig. 9. (In color) Stress contours from the FEM model (a-c) and the corresponding stress maps (d-f) from the MD model (corresponding to Fig. 5(b) at $3.75 \mathrm{GPa}$ prestress) for $\sigma_{x x}, \sigma_{y y}$, and $\sigma_{x y}$ stress components. The stress-level colors are defined in the same way as in Fig. 8. Formations (1)-(6) correspond to those indicated in Fig. 5(b). 
To be submitted to: Journal of the Mechanics and Physics of Solids

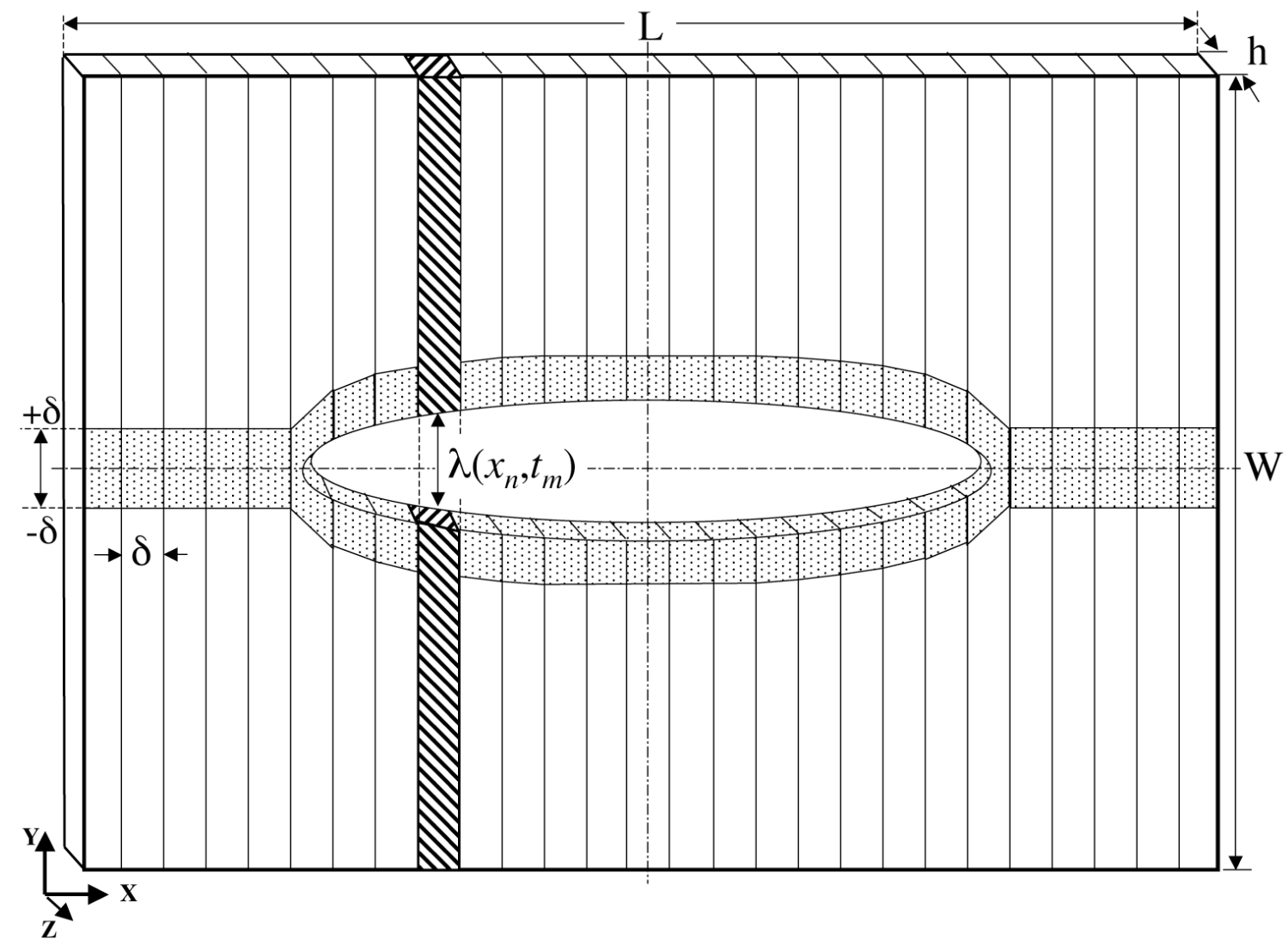

Fig. 10. Schematic diagram of the slicing of the system volume in the MD simulation and defining the representative regions for extracting the parameters for the cohesive-zone interface elements in a continuum simulation. 
To be submitted to: Journal of the Mechanics and Physics of Solids

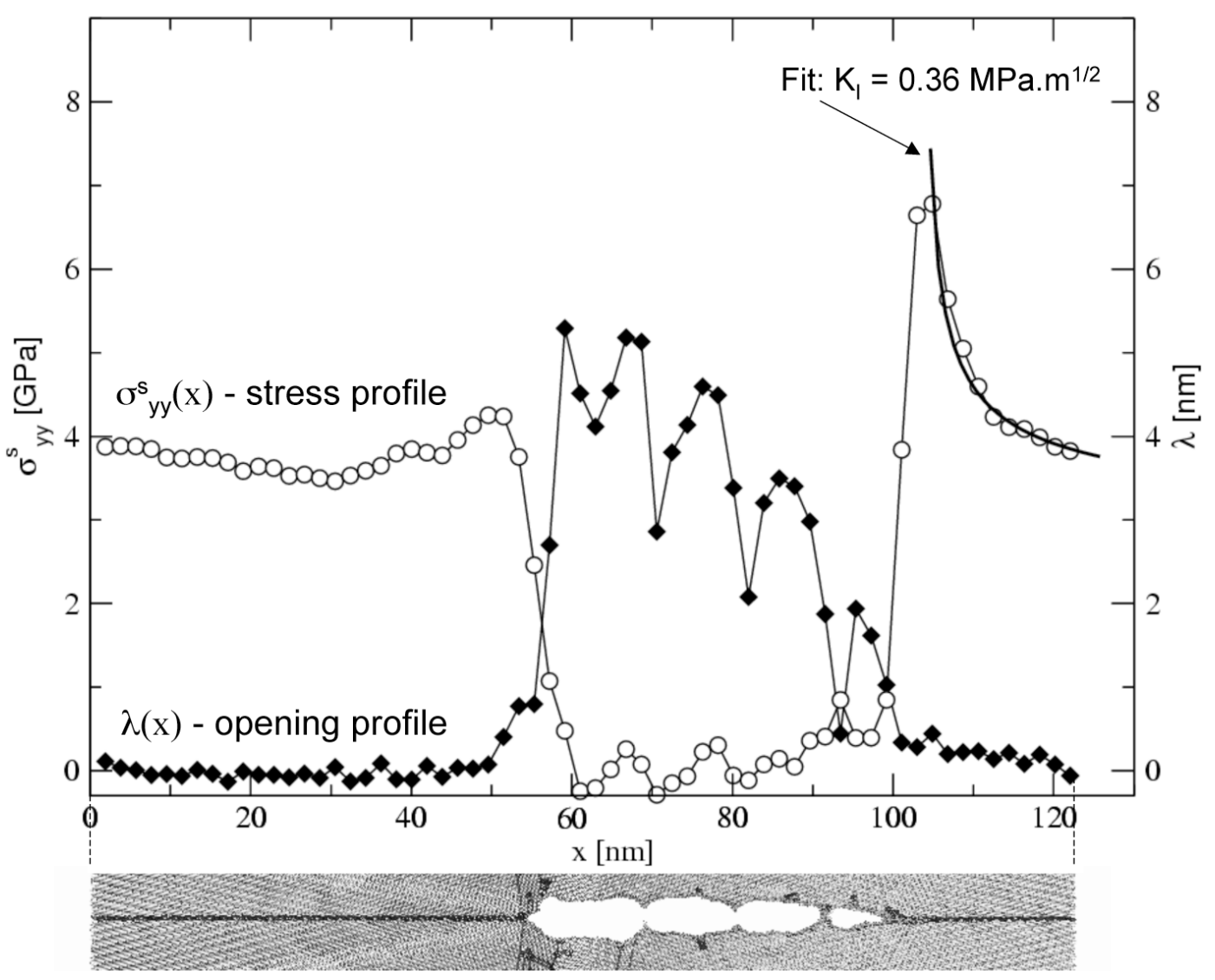

Fig. 11. Stress and opening profiles extracted along the crack growing for $123 \mathrm{ps}$ in a system prestressed at $4.25 \mathrm{GPa}$ hydrostatic load. The corresponding snapshot of the crack is shown at the bottom. 
To be submitted to: Journal of the Mechanics and Physics of Solids
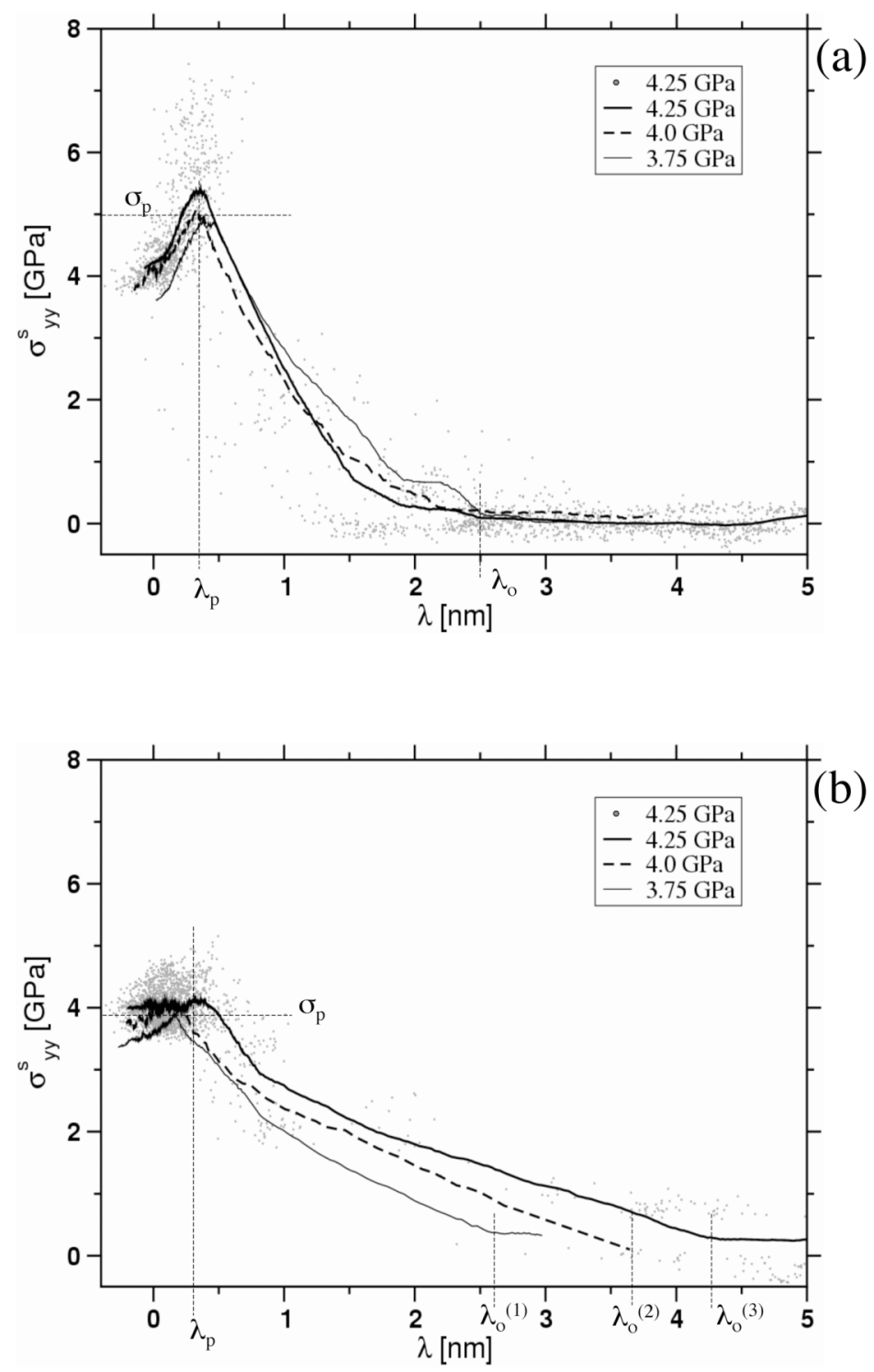

Fig. 12. Surface stress vs. crack opening curves $\sigma_{\text {yy }}^{\mathrm{s}}(\lambda)$ characterizing the propagation of the cleavage tip in the $+x$ direction (a) and in the $-x$ direction (b) for three preloads. The dots are the individual measurements for 4.25 GPa hydrostatic load simulation, from which an average was taken to produce the corresponding $\sigma_{\mathrm{yy}}^{\mathrm{s}}(\lambda)$ curve (see text). The curves for 3.75 and $4.0 \mathrm{GPa}$ are produced in a similar way. 
To be submitted to: Journal of the Mechanics and Physics of Solids

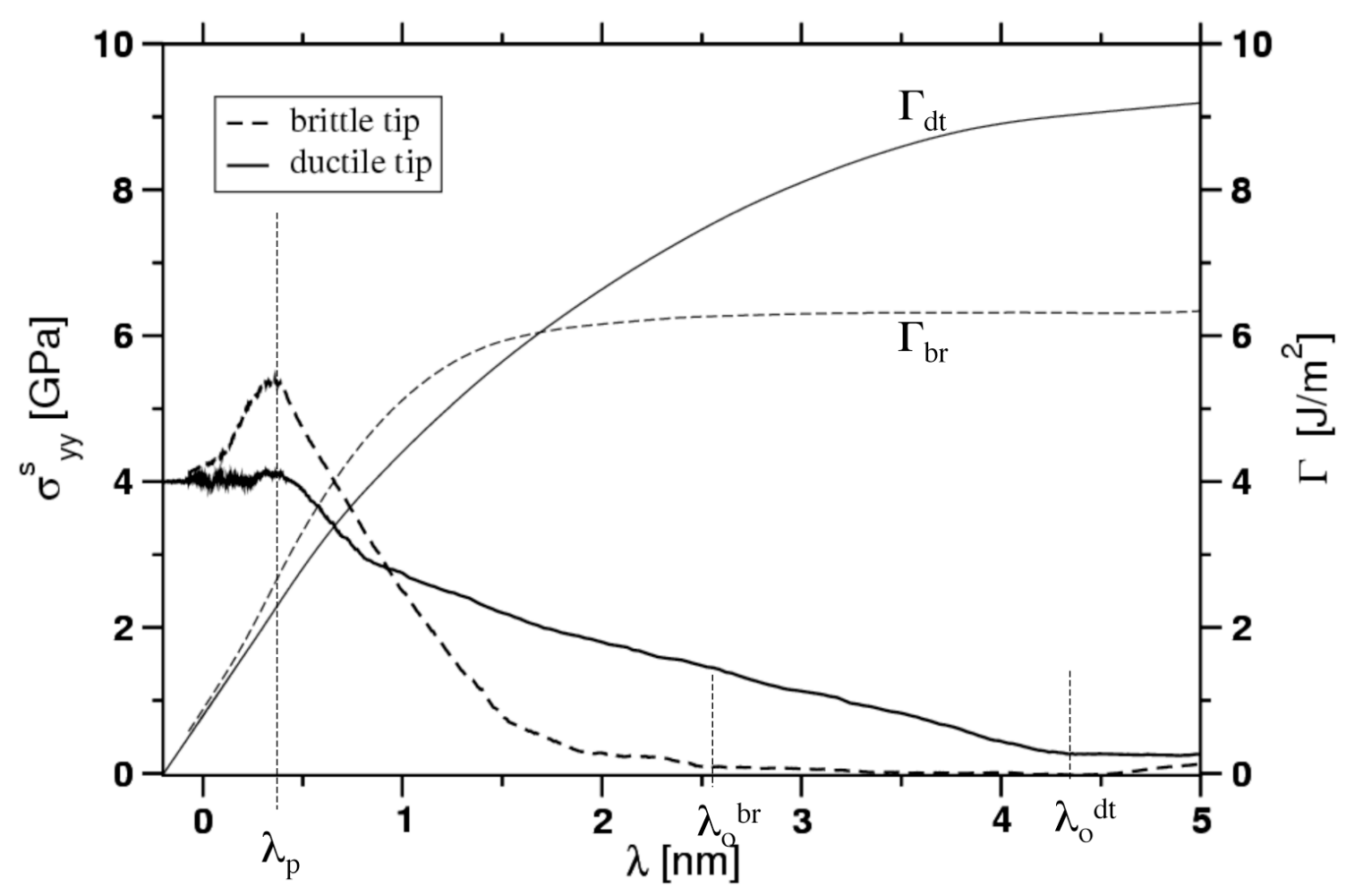

Fig. 13. Traction-displacement relationship $\sigma_{\mathrm{yy}}^{\mathrm{s}}(\lambda)$ and the corresponding work of separation $\Gamma(\lambda)$ for the case of $4.25 \mathrm{GPa}$ prestress compared for the brittle $(+x)$ and the twinning $(-x)$ tip. 